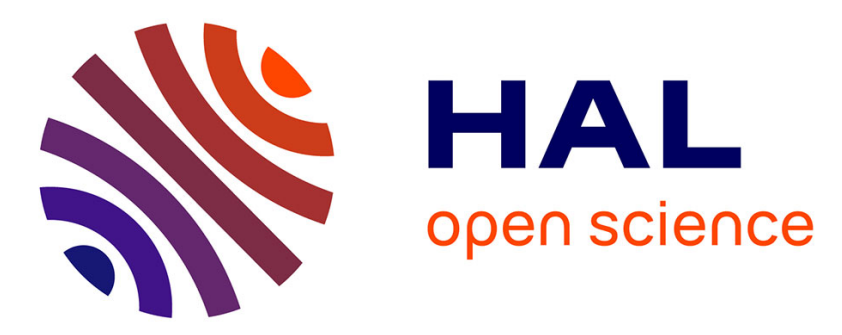

\title{
Méthodes expérimentales d'étude de la relaxation d'une vapeur alcaline orientée optiquement
}

Marie-Anne Bouchiat, Françoise Grossetête

\section{To cite this version:}

Marie-Anne Bouchiat, Françoise Grossetête. Méthodes expérimentales d'étude de la relaxation d'une vapeur alcaline orientée optiquement. Journal de Physique, 1966, 27 (5-6), pp.353-366. 10.1051/jphys:01966002705-6035300 . jpa-00206414

\section{HAL Id: jpa-00206414 https://hal.science/jpa-00206414}

Submitted on 1 Jan 1966

HAL is a multi-disciplinary open access archive for the deposit and dissemination of scientific research documents, whether they are published or not. The documents may come from teaching and research institutions in France or abroad, or from public or private research centers.
L'archive ouverte pluridisciplinaire HAL, est destinée au dépôt et à la diffusion de documents scientifiques de niveau recherche, publiés ou non, émanant des établissements d'enseignement et de recherche français ou étrangers, des laboratoires publics ou privés. 


\title{
MÉTHODES EXPÉRIMENTALES D'ÉTUDE DE LA RELAXATION D'UNE VAPEUR ALCALINE ORIENTÉE OPTIQUEMENT
}

\author{
Par Marie-Anne BOUChiAT et Françoise Grossetête, \\ Laboratoire de Physique de l'E. N. S., Paris, 5e.
}

\begin{abstract}
Résumé. - Une nouvelle méthode optique d'étude de la relaxation d'une vapeur alcaline orientée optiquement est décrite : le faisceau lumineux pompant $\mathrm{F}^{\mathfrak{D}}$ (destiné à préparer le système atomique dans un état hors de l'équilibre de Boltzmann) est distinct du faisceau détecteur $\mathrm{F}^{d}$. Les caractéristiques de $\mathrm{F}^{d}$ (polarisation, profil spectral) déterminent la nature de l'observable $Q$ que la mesure de la lumière absorbée sur $\mathrm{F}^{d}$ permet d'étudier. $\mathrm{F}^{d}$ est allumé en permanence et est choisi suffisamment peu intense pour ne pas perturber l'évolution du système sous le seul effet de la relaxation : il permet d'observer directement la courbe"de relaxation de $\langle Q\rangle$ lors de la coupure de $\mathrm{F}^{p}$.

Cette méthode présente de grands avantages par rapport à celle utilisant la séquence de relaxation dans le noir de Franzen. Elle permet aussi de réaliser des expériences nouvelles. Nous décrivons sa mise en œuvre sur une vapeur de $\mathrm{Rb}$ pour l'étude de la relaxation de $\left\langle S_{z}\right\rangle$, et de celle de $\langle\mathbf{S}$.I $\rangle$ en présence ou en l'absence d'une polarisation électronique globale $\left\langle S_{z}\right\rangle$. La relaxation de $\langle\mathbf{S}$.I $\rangle$ en l'absence de $\left\langle S_{z}\right\rangle$ peut s'étudier de la même manière pour d'autres alcalins que Rb et pour des mélanges d'alcalins. Ceci permet de mesurer le rapport des sections efficaces d'échange de spin entre atomes alcalins sans avoir à connaittre la valeur absolue des tensions de vapeur. En particulier, nos mesures indiquent que les sections efficaces ${ }^{85} \mathrm{Rb}$-Cs, ${ }^{87} \mathrm{Rb}$-Cs et Cs-Cs sont égales à la précision de nos déterminations.
\end{abstract}

\begin{abstract}
An optical method is described for studying the relaxation of alkali atoms polarized by optical pumping. Two light beams are used : the first $\mathrm{F}^{\boldsymbol{p}}$ for pumping, and the second $\mathrm{F}^{d}$ for detection. The amount of light absorbed per unit time on $\mathrm{F}^{d}$ determines the observable $Q$ whose relaxation is actually measured : $Q$ depends on the polarization and spectral distribution of $\mathrm{F}^{d}$. $\quad \mathrm{F}^{d}$ is kept on continuously and its intensity is chosen to be low enough not to disturb the relaxation of $\langle Q\rangle$ : then the evolution of $\langle Q\rangle$ when $\mathrm{F}^{p}$ is suddenly turned off can be observed directly.

This new method compared to Franzen's (relaxation in the dark) brings many advantages and new types of experiments become feasible. We describe how it has been applied to the case of $\mathrm{Rb}$ vapor to study the relaxation of $\left\langle S_{z}\right\rangle$ and the relaxation of $\langle\mathbf{S}$.I $\rangle$ in the absence or the presence of $\left\langle S_{z}\right\rangle$. The relaxation of $\langle$ S.I $\rangle$ in the absence of $\left\langle S_{z}\right\rangle$ can be studied similarly for other alkali atoms (pure isotopes or mixtures of isotopes). It is thus possible to measure the ratio between spin exchange cross sections of alkali atoms. We have obtained the following result : ${ }^{85} \mathrm{Rb}-\mathrm{Cs},{ }^{87} \mathrm{Rb}-\mathrm{Cs}$ and $\mathrm{Cs}-\mathrm{Cs}$ exchange cross sections are equal within the accuracy of our measurements.
\end{abstract}

Introduction. - Les méthodes d'étude de la relaxation que nous envisageons dans le présent article ont les caractères suivants :

- On prépare le système (vapeur d'atomes alca. lins à basse pression) dans un état initial qui n'est pas celui de l'équilibre de Boltzmann, puis on le laisse évoluer sous l'action du seul processus de relaxation auquel on s'intéresse (collisions contre la paroi, contre un gaz étranger, collisions d'échange, etc...).

- Tant pour préparer le système que pour suivre son évolution, on utilise des méthodes optiques.

La préparation du système utilise le pompage optique [1], [2] par un premier faisceau lumineux $\mathrm{F}^{p}$ d'intensité $i^{p}$. Ce faisceau a une composition spec- trale et une polarisation choisies pour réaliser l'état initial à partir duquel se produit la relaxation. Si l'on coupe $\mathrm{F}^{p}$, on peut en effet laisser le système évoluer " dans le noir " sous le seul effet de cette dernière. On suit l'évolution à l'aide d'un deuxième faisceau, le faisceau détecteur $\mathrm{F}^{d}$ d'intensité $i^{d}$; ses caractéristiques (profil spectral, polarisation) déterminent l'observable $Q$ sur laquelle porte la mesure qui concerne essentiellement la quantité de lumière $L_{\mathrm{A}}$ absorbée par unité de temps sur $\mathrm{F}^{d}[3]$.

Méthode 1. - Une première méthode, due à Franzen [4], consiste à suivre l'évolution de $\langle Q\rangle$ en allumant $F^{d}, \theta$ secondes après la coupure de $F^{p}$ et en mesurant $\langle Q\rangle$ à cet instant. On recommence la séquence en faisant varier $\theta$. 
Il est possible de faire coïncider les faisceaux $\mathrm{F}^{d}$ et $\mathrm{F}^{\mathfrak{p}}$. C'est sous cette dernière forme que la méthode d'étude de la relaxation dans le noir a été initialement proposée ( $f g .1)$ et fréquemment exploitée par la suite.

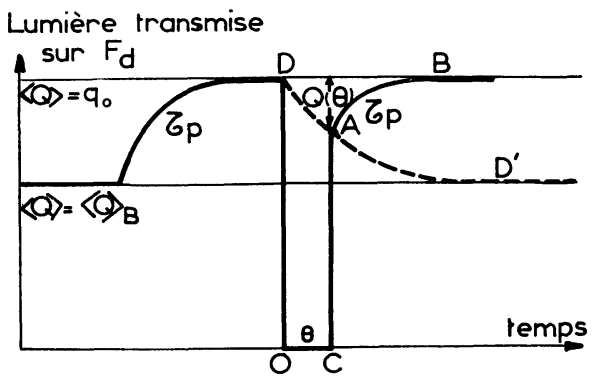

Fig. 1. - Méthode 1: Dans une première phase, on illumine la vapeur avec une forte intensité $i^{p}$ de manière à préparer le système atomique dans un état où $\langle Q\rangle=q_{0}$ est très différent de $\langle Q\rangle_{\text {B }}$ : l'intensité transmise croît suivant une courbe d'allure exponentielle de "pseudo ) constante de temps $\tau_{p}$. A un instant (pris comme zéro) on coupe brusquement $\mathrm{F}^{\boldsymbol{p}}$, l'intensité de la lumière transmise (courbe continue) tombe à zéro. Pendant la coupure de $\mathrm{F}^{p}$, $\langle Q\rangle$ relaxe dans le noir et décroît suivant la courbe pointillée $\mathrm{DD}^{\prime}$ que nous désirons connaître. A l'instant $\theta$, lorsque $\mathrm{F}^{p}$ est rétabli, le signal optique part de $\mathrm{A}$ et l'on observe à partir de ce point la courbe de pompage optique caractérisée par le temps $\tau_{p}$. En faisant varier $\theta$, A décrit la courbe de relaxation $\mathrm{DD}^{\prime}$.

Méthode 2. - Nous nous proposons de décrire ici, en détail, une méthode différente $: \mathrm{F}^{d}$ est distinct de $\mathrm{F}^{p}$ et choisi suffisamment peu intense pour qu'il ne perturbe pas de façon détectable l'évolution du système sous le seul effet de la relaxation. $\mathrm{F}^{d}$ est allumé en permanence. La lumière absorbée $L_{A}$ sur $\mathrm{F}^{d}$ mesure la valeur initiale de l'observable, $\langle Q\rangle=q_{0}$; après coupure de $\mathrm{F}^{p}, L_{\mathrm{A}}$ permet de suivre la courbe de relaxation de $\langle Q\rangle$. Au lieu d'être construite point par point, la courbe de relaxation "dans le noir " peut ainsi s'obtenir directement au cours d'une séquence unique, la relaxation lumineuse due à $\mathrm{F}^{d}$ étant totalement négligeable (fig. 2).

Il nous paraît souhaitable de rappeler tout d'abord quelques faits relatifs à la relaxation d'atomes alcalins $(\S \mathrm{I})$. Nous comparerons ensuite les deux méthodes ( $\S$ II), nous verrons que la seconde présente de multiples avantages. Enfin nous décrirons sa réalisation pour l'étude de la relaxation de $\langle\mathbf{S}$.I $>$ sur une vapeur de $\mathrm{Rb}$ ou de $\mathrm{Cs}(\S \mathrm{III})$, et de $\left.<\mathrm{S}_{z}\right\rangle$ sur une vapeur de $R b(\S I V)$.

I. Rappels concernant la relaxation d'atomes alcalins. - Nous groupons dans ce paragraphe quelques résultats qui permettent de bien comprendre la nature des problèmes destinés à être étudiés par les méthodes que nous envisageons dans cette publication. Par la suite, nous nous référerons souvent à ces résultats.

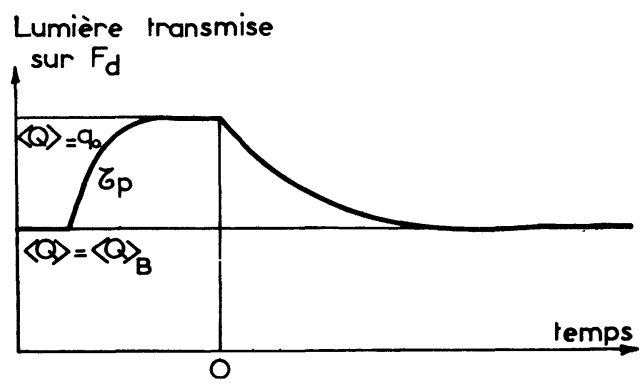

FIg. 2. - Méthode 2 : Dans une première phase, on prépare le système atomique dans un état où $\langle Q\rangle=q_{0}$ à l'aide d'un faisceau $\mathrm{F}^{p}$ distinct de $\mathrm{F}^{d}$, d'intensité $i^{p}$ : l'intensité transmise sur $\mathrm{F}^{d}$ croît suivant la courbe de pompage optique caractérisée par le temps $\tau_{\nu}$. A un instant (pris comme 0 ), on coupe $\mathrm{F}^{p}$, le signal optique sur $\mathrm{F}^{d}$ permet l'observation directe de la courbe de relaxation de $\langle Q\rangle$.

1) Relaxation sous L'effet d'une interacTION DÉSORIENTATRICE ALÉATOIRE FAIBLE DE TYPE MAGNÉTIQUe. - On suppose que cette interaction est traduite par un hamiltonien aléatoire de la forme $\mathcal{H}_{1}(t)=\gamma_{\mathbf{S}} \mathbf{S} . \mathbf{H}(t)$; il n'agit que sur le spin électronique $\mathbf{S}$ de l'alcalin, $\mathbf{H}(t)$ est un champ magnétique aléatoire satisfaisant au critère de moyennage par le mouvement. Ces conditions se trouvent par exemple réalisées expérimentalement pour la relaxation d'atomes de $\mathrm{Rb}$ par collisions sur une paroi enduite de paraffine ou par collisions sur des molécules de gaz tampon [5].

Le calcul théorique [6] montre que certaines observables $Q_{i}$ relaxent exponentiellement vers leur valeur moyenne à l'équilibre de Boltzmann $\left.<Q_{i}\right\rangle_{B}$ avec une constante de temps unique $T_{i}$ (quel que soit l'état initial du système atomique). Si on se limite aux grandeurs longitudinales, il y a $4 I+1$ observables $Q_{i}, I$ étant le spin nucléaire de l'alcalin. En champ faible, la nature de ces observables est indépendante du temps de corrélation de l'interaction ; citons en particulier :

$\left\langle\mathrm{I}_{z}\right\rangle$, polarisation nucléaire longitudinale.

$\left\langle Q_{\mathrm{e}}\right\rangle$, combinaison linéaire de $\left\langle I_{z}\right\rangle$ et de $<S_{z}>$ (polarisation électronique longitudinale) définie par :

$$
<Q_{\mathrm{e}}>=<S_{z}>-\frac{2}{(2 I+1)^{2}-2}<I_{z}>
$$

$<$ S.I $>$, écart par rapport à l'équilibre de Boltzmann de la différence de populations entre niveaux hyperfins. Si l'on dénote par $P_{F_{ \pm}}$les opéra- 
teurs de projection sur les états $F_{ \pm}=I \pm 1 / 2$, on a la relation :

$$
\begin{aligned}
<\mathbf{S} . \mathbf{I}>= & \frac{2 I+1}{4}\left[<P_{F_{+}}>-<P_{F_{-}}>\right. \\
& \left.-\left(<P_{H_{+}}>_{B}-<P_{F_{-}}>_{B}\right)\right] \quad(2 . a) \\
= & \frac{1}{4 g_{F}},\left[<P_{H_{+}}>-<P_{F_{-}}>-g_{F}^{\prime}\right] .
\end{aligned}
$$

dans laquelle $g_{F}^{\prime}=1 /(2 I+1)$.

Nous désignons par $T_{n}, T_{\mathrm{e}}, T_{H}$ les constantes de temps associées respectivement à $\left\langle I_{z}\right\rangle,\left\langle Q_{\mathrm{e}}\right\rangle$, $<$ S.I $>$.

Il résulte également du calcul théorique qu'à toute grandeur longitudinale peut être associé un opérateur $Q$ qui peut s'écrire de manière unique :

$$
Q=\sum_{i} a_{i} Q_{i} \text {. }
$$

L'évolution dans le temps de $\langle Q\rangle$ est donc de la forme :

$$
\frac{\mathrm{d}}{\mathrm{d} t}<Q>=\sum_{i} a_{i} \leq Q_{i}>-<Q_{i}>_{B} .
$$

Par exemple la relaxation de $\left\langle S_{z}>\right.$ est décrite par l'équation :

$$
\begin{aligned}
\frac{\mathrm{d}}{\mathrm{d} t}<S_{z}>=- & \frac{\left\langle Q_{\mathrm{e}}\right\rangle}{T_{\mathrm{e}}} \\
& -\frac{2}{(2 I+1)^{2}-2} \cdot \frac{\leq I_{z}>}{T_{n}} .
\end{aligned}
$$

Elle fait intervenir les constantes de temps $T_{\mathrm{e}}$ et $T_{n}$ qui caractérisent la relaxation exponentielle de $\left\langle Q_{\mathrm{e}}\right\rangle$ et de $\left\langle I_{\mathrm{z}}\right\rangle$. On voit sur l'équation (5) que s'il s'agit de mesurer simplement $T_{\mathrm{e}}$ et $T_{n}$, il importe peu que la mesure soit effectuée sur $\left\langle S_{z}\right\rangle$ ou sur une combinaison linéaire de $\left\langle S_{z}\right\rangle$ et de $\left\langle I_{z}\right\rangle$. Nous ferons usage de cette remarque plus tard.

2) Relaxation sous l'effet des seules colliSIONS D'ÉCHANGE DE SPIN ENTRE ATOMES ALCALINS DIFFÉRENTS. - Le cas envisagé est celui d'une certaine catégorie d'atomes alcalins A susceptibles d'interagir par collisions d'échange avec des atomes alcalins différents B. Le calcul théorique [7] aboutit à la conclusion suivante : si la polarisation électronique moyenne de $B$ est maintenue nulle $\left(\left\langle S_{z}^{B}\right\rangle=0\right)$, l'équation d'évolution de n'importe quelle observable $Q$ prend la même forme que dans le cas précédent (cf. éq. 4). Les observables $Q_{i}$ sont les mêmes. On peut, en particulier, définir des constantes de temps $T_{n}^{\prime}, T_{e}^{\prime}, T_{H}^{\prime}$ pour l'évolution de $\left\langle I_{z}\right\rangle$, $\left\langle Q_{\mathrm{e}}\right\rangle$ et $<$ S.I $>$; elles sont inversement proportionnelles à la fréquence de collision d'un atome A sur les atomes $B$.
3) Relaxation sous h'effet des collisions D'ÉCHANGE ENTRE ATOMES ALCALINS IDENTIQUES. Le problème correspondant a été traité du point de vue théorique [8].

a) Relaxation de $\left\langle S_{\mathrm{z}}\right\rangle$ et de $\langle\mathbf{S}$.I $\rangle$ en présence de $\left\langle S_{z}\right\rangle$ : Lorsque le système atomique est préparé dans un état initial où il possède une polarisation électronique $\left\langle S_{z}\right\rangle_{0}$ non nulle, l'état d'équilibre atteint sous le seul effet des collisions d'échange entre atomes identiques n'est pas l'équilibre de Boltzmann (chaque collision conserve le moment angulaire total : $\left\langle F_{z}\right\rangle$ est inchangé). Pour traiter un cas réaliste, il faut tenir compte d'une seconde cause de relaxation qui fait tendre le système vers l'équilibre de Boltzmann, par exemple la relaxation due à une interaction aléatoire faible, de type magnétique. Le problème se pose alors de la manière suivante : calculer les modifications apportées par les collisions d'échange aux résultats du paragraphe I-1. Les conclusions concernant $\left\langle S_{z}\right\rangle$ et $\langle$ S.I $\rangle$ sont les suivantes :

- Relaxation de $\left\langle S_{z}\right\rangle$ : Nous n'avons jamais pu détecter une influence des collisions d'échange lorsque les mesures sont faites sur un isotope pur [9]. La théorie des collisions d'échange entre atomes identiques permet d'expliquer ces observations [8].

- Relaxation de $<\mathbf{S} . \mathbf{I}>$ en présence de $\left\langle S_{z}\right\rangle$ : L'équation d'évolution sous l'effet simultané des collisions d'échange entre atomes identiques et des collisions satisfaisant aux critères du paragraphe I-1 est la suivante (en supposant les aimantations transversales nulles) :

$$
\begin{aligned}
\frac{\mathrm{d}}{\mathrm{d} t}<\mathbf{S} . \mathbf{I} & >-\frac{1}{T_{H}}<\mathbf{S . I}> \\
& \quad-\frac{1}{T_{\mathrm{e} H}}\left[<\mathbf{S . I}>-<S_{z}><I_{z}>\right]
\end{aligned}
$$

$T_{\mathrm{e} H}$ est le temps qui sépare deux collisions d'échange [7].

L'équation (6) traduit un couplage entre $<$ S.I $>$ et $\left\langle S_{z}\right\rangle$ sous l'effet des collisions d'échange. Nous venons de voir que $\left\langle S_{z}\right\rangle$ évolue avec les constantes de temps $T_{n}$ et $T_{\mathrm{e}},\left\langle I_{z}\right\rangle$ évolue avec la constante de temps $T_{n}$, donc

$$
<S_{z}><I_{z}>=\alpha \mathrm{e}^{-t / T}+\beta \mathrm{e}^{-t / T^{\prime}}
$$

où

$$
1 / T=2 / T_{n} \quad \text { et } \quad 1 / T^{\prime}=1 / T_{n}+1 / T_{\mathrm{e}} .
$$

Par suite, l'évolution de $<$ S.I $>$ est caractérisée par trois constantes de temps $T, T^{\prime}$ et $T_{1 H}$ $\left(1 / T_{1 H}=1 / T_{H}+1 / T_{\mathrm{e} H}\right)$.

Dans une cellule recouverte d'un enduit, et en l'absence de gaz, $T$ et $T^{\prime}$ sont assez voisins pour le rubidium (on déduit des valeurs $T_{\mathrm{e}}$ et $T_{n}$ mesurées sur un enduit $\mathrm{CD}_{2}$, que $T / T^{\prime}$ vaut 2,1 pour ${ }^{87} \mathrm{Rb}$ et 2,8 pour ${ }^{85} \mathrm{Rb}$ ) et, dans les conditions usuelles, $T_{1 H}$ diffère peu du temps d'échange $T_{\mathrm{e} H}$, très court 
devant les constantes de temps $T, T^{\prime}, T_{H}$ caractéristiques de la relaxation sur la paroi.

D'autre part, le pompage optique en présence de relaxation sous l'effet des diverses collisions conduit à un état stationnaire tel que :

$$
<\mathbf{S . I}>_{0} \simeq<S_{z}>_{0}<I_{z}>_{0}
$$

(nous avons fait des calculs sur machine I. B. M. le prouvant). C'est à partir de cet état que se fait la relaxation dans le noir que nous étudions. Il en résulte que le poids de la constante de temps courte $T_{1 H}$ est très faible comparé à celui des deux autres ; pratiquement, on ne voit pas $T_{1 H}$. Les deux autres constantes de temps étant voisines, il est difficile de les distinguer l'une de l'autre et la relaxation de $\langle$ S.I $\rangle$ se fait avec une "pseudo "constante de temps $\tau\left(T^{\prime}<\tau<T\right)$; la valeur de $\tau$ dépend des poids relatifs de $T$ et $T^{\prime}$. On peut montrer que lorsque la polarisation initiale $\left\langle S_{z}>_{0}\right.$ diminue, $\alpha / \beta$ augmente, $\tau$ s'allonge et tend vers $T_{n} / 2$

$\beta)$ Relaxation de $\langle\mathbf{S}$.I $\rangle$ en absence de $\left\langle S_{z}\right\rangle$ : $\mathrm{La}$ relaxation d'un système atomique qui ne possède pas de polarisation électronique globale $\left(\left\langle S_{z}\right\rangle_{0}=0\right)$ est un problème plus simple. On démontre que, en l'absence de $\left\langle S_{z}\right\rangle$, $\langle$ S.I $\rangle$ évolue exponentiellement vers l'équilibre de Boltzmann sous le seul effet des collisions d'échange avec la constante de temps $T_{\mathrm{e} H}$. Cette dernière est inversement proportionnelle au nombre $N_{\alpha}$ d'atomes par $\mathrm{cm}^{3}$ :

$$
\frac{1}{T_{\mathrm{e} H}}=4 N_{\alpha} \pi \sigma_{\alpha \alpha}^{2} \sqrt{R T / \pi M_{\alpha}}
$$

$\pi \sigma_{\alpha \alpha}^{2}$ étant la section efficace d'échange entre atomes alcalins identiques $\alpha$.

En ce qui concerne l'évolution de $\langle\mathbf{S . I}\rangle$ en l'absence de $\left\langle S_{z}\right\rangle$ on voit donc que les collisions d'échange entre atomes identiques ou différents produisent le même effet.

Si l'on tient compte des résultats énoncés au paragraphe I-1, on voit que sous l'effet simultané de la relaxation sur la paroi et par collisions d'échange, la relaxation de $\left\langle\right.$ S.I $>$ en l'absence de $\left\langle S_{z}\right\rangle$ est encore exponentielle de constante de temps $T_{1 H}$ :

$$
1 / T_{1 \mathrm{H}}=1 / T_{\mathrm{H}}+1 / T_{\mathrm{e} H}
$$

$1 / T_{1 H}$ est représenté par une droite en fonction de $N_{\alpha}$. Ce résultat a été vérifié expérimentalement. On peut déduire $\pi \sigma_{\alpha \alpha}^{2}$ de la pente de cette droite et $1 / T_{H}$ de son ordonnée à l'origine [10].

II. Comparaison des deux méthodes. - 1) Pour être valable, la méthode 2 exige que l'on choisisse soigneusement $i^{d}$. Étant donnée une observable dont l'évolution sous le seul effet de la relaxation est décrite par l'équation (4), on peut montrer [11] que si $F^{d}$ est suffisamment peu intense, l'évolution de
$<Q>$ sous l'effet simultané de la relaxation et de $F^{d}$ est décrite par :

$$
\frac{\mathrm{d}<Q>}{\mathrm{d} t}=-\sum_{i} a_{i} \frac{\left\langle Q_{i}>-<Q_{i}^{0}>\right.}{\tau_{i d}}
$$

$<Q_{i}^{0}>$ étant la valeur moyenne de l'observable $Q_{i}$ à l'état stationnaire en présence de $\mathrm{F}^{d}$; la constante de temps $\tau_{i d}$ qui tend vers $T_{i}$ quand $i^{d}$ tend vers 0 , peut s'écrire :

$$
\frac{1}{\tau_{i d}}=\frac{1}{\bar{T}_{i}}+\frac{k_{i}}{T_{d}}
$$

$k_{i}$ est un coefficient numérique qui se calcule explicitement pour chaque observable $Q_{i}$ connaissant les caractéristiques de $\mathrm{F}^{d} ; T_{d}$ représente le temps moyen qui sépare l'absorption successive de deux photons de $\mathrm{F}^{d}$ par le même atome et est inversement proportionnel à $i^{d}$.

La méthode 2 n'est donc valable que si, à la précision expérimentale près, (4) et (9) représentent la même équation d'évolution, c'est-à-dire si l'on choisit $i^{d}$ suffisamment faible pour que la condition

$$
T_{d} \gg k_{i} T_{i}
$$

soit vérifiée, pour toutes les constantes de temps $T_{i}$ qui participent à la relaxation de $\langle Q\rangle$.

Le résultat se généralise facilement au cas où l'évolution de $\langle Q\rangle$ n'est pas régie par une équation différentielle linéaire (cas des collisions d'échange entre atomes identiques), $T_{d}$ devant être dans ce cas plus long que la plus longue des " pseudo" constantes de temps qui participent à la relaxation de $\langle Q\rangle$.

Par ailleurs $i^{d}$ ne peut être choisi trop faible car, à bande passante donnée, le rapport signal sur bruit $S / B$ diminue avec $i^{d}$ comme $\sqrt{i^{d}}$ (pour de faibles intensités détectrices, la cause essentielle de bruit est le bruit Shott, proportionnel à $\sqrt{i^{d}}$, engendré au niveau de la photocathode).

Le rapport $S / B$ est d'autant meilleur que la bande passante est plus étroite. Pour étudier les séquences des figures 1 et 2 , on obtient une amélioration considérable en utilisant un filtre passe-bas dont la fréquence de coupure est grande par rapport aux fréquences les plus grandes présentes dans le spectre à analyser. Il est clair qu'à cet égard la séquence du type 2 présente des avantages considérables sur celle du type 1 parce qu'elle peut être transmise sans déformation avec une bande passante beaucoup plus étroite : on a en effet affaire à une somme d'exponentielles de constantes $\tau_{i d}$ très voisines de $T_{i}$ (puisque $T_{d}$ est très long). Dans le cas 1 au contraire, les transitoires font intervenir des temps $\tau_{i_{p}}$ qui sont de l'ordre de $T_{z}$, puisque l'intensité $i^{p}$ de pompage est prise aussi grande que possible. La situation est même encore plus défavorable, puisque la mesure dans la séquence 1 concerne 
la discontinuité $A$. Il est crucial que la brisure A dans la séquence DOCAB puisse s'observer nettement, ce qui nécessite une bande passante beaucoup plus large que dans l'autre cas.

En résumé, la méthode 2 présente le désavantage d'utiliser nécessairement une intensité détectrice faible, mais elle permet d'un autre côté l'utilisation de bandes passantes beaucoup plus étroites : l'expérience montre qu'en ce qui concerne le rapport $S / B$, il y a pratiquement compensation. En outre, d'autres facteurs que l'intensité globale détectrice $i^{d}$ influent sur la sensibilité de la détection; en les analysant, nous verrons que la méthode 2 présente de grands avantages.

2) Par la méthode 1, la construction de la courbe de relaxation (surtout lorsque celle-ci est la superposition de plusieurs exponentielles) nécessite un très grand nombre de séquences correspondant à des valeurs de $\theta$ différentes; outre la patience de l'expérimentateur, la méthode 1 exige une bonne stabilité de l'intensité émise par la lampe et de la tension de vapeur étudiée pendant tout le temps nécessaire aux mesures.

La méthode 2 étant beaucoup plus rapide est aussi bien moins sensible aux dérives diverses (lampes, etc...).

3) En outre, une séquence du type de celle qu'utilise la méthode 2 peut être facilement répétée un très grand nombre de fois. Dans le cas des signaux petits, ceci permet une amélioration considérable du rapport $S / B$ par l'emploi d'un procédé de moyennage utilisant le principe des analyseurs multicanaux [12] (nous avons utilisé le "Enhancetron"). Au contraire, la séquence réalisée dans la méthode 1 est complètement inadaptée à une telle exploitation des résultats expérimentaux.

4) La coupure de $\mathrm{F}^{d}$ (ou de $\mathrm{F}^{p}$ si $\mathrm{F}^{d}$ et $\mathrm{F}^{p}$ coïncident) exigée par la méthode 1 est à l'origine de la difficulté suivante : lorsqu'on rétablit la lumière, les photomultiplicateurs infrarouges présentent de grands effets de fatigue [13] dont les constantes de temps sont souvent du même ordre que celles des transitoires de pompage optique à étudier. Des précautions sont donc à prendre pour éliminer ces effets ou pour en tenir compte sous forme de correction apportée aux résultats expérimentaux.

La méthode 2 permet d'éviter la coupure de $\mathrm{F}^{d}$, mais elle ne peut éviter celle de $\mathrm{F}^{p}$ et le photomultiplicateur $\mathrm{PM}_{d}$ placé sur $\mathrm{F}^{d}$ détecte une certaine variation d'intensité $\Delta i^{p}$, due à ce qu'une faible fraction $\mathrm{du}$ faisceau pompant très intense et très ouvert est susceptible d'atteindre $\mathrm{PM}_{d}$ après réflexion et diffusion sur le verre de la cellule $C_{1}$ et de son four (fig. 3). Comme $\mathrm{F}^{d}$ est très peu intense (suivant le temps de relaxation à mesurer, $i^{d}$ est 50 à 500 fois plus faible que $\left.i^{p}\right), \Delta i^{n}$ n'est pas du tout négligeable devant $i^{d}$; dans certains cas, cette variation rapide $\Delta i^{p}$ associée à la coupure de $\mathrm{F}^{p}$ peut même produire sur $\mathrm{PM}_{d}$ qui est très sensible, un

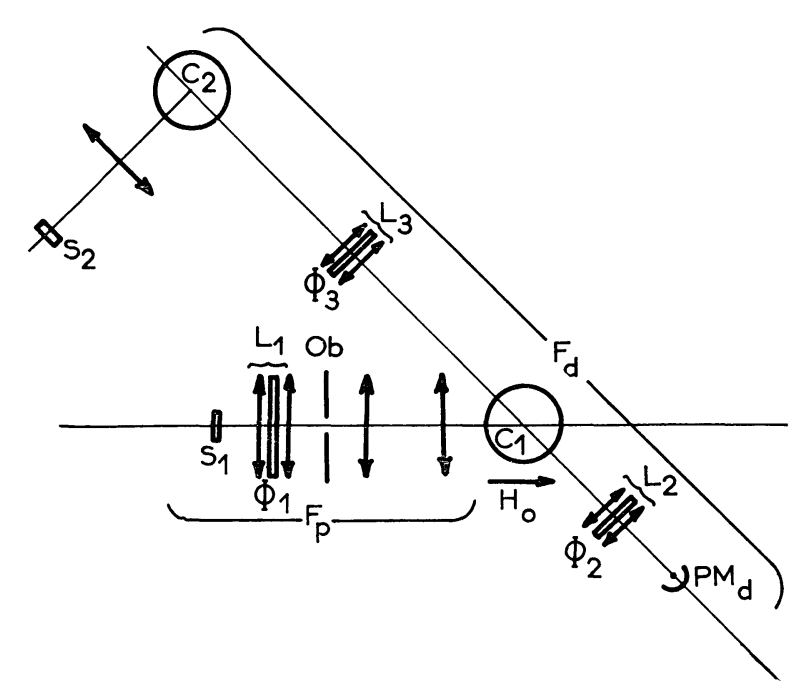

FIG. 3. - Schéma du montage expérimental. Le faisceau pompant $\mathrm{F}^{p}$ peut être coupé en manœuvrant l'obturateur (Ob). Le faisceau détecteur $\mathrm{F}^{d}$ (50 à 500 fois moins intense) est allumé en permanence.

effet transitoire similaire à celui auquel on s'intéresse. Pour éliminer cet inconvénient grave, on choisit pour $\mathrm{F}^{p}$ et $\mathrm{F}^{d}$ des longueurs d'onde différentes. $\mathrm{F}^{p}$ traverse un filtre interférentiel $\Phi_{1}$ laissant passer l'une des deux raies $D, D_{1}$ (ou $D_{2}$ ); sur $\mathrm{F}^{d}$, on place juste avant $\mathrm{PM}_{d}$ un second filtre interférentiel $\Phi_{2}$ ne laissant passer que l'autre composante, soit $D_{2}$ (ou $D_{1}$ ). Si les filtres interférentiels sont de bonne qualité, $\mathrm{PM}_{d}$ devient ainsi pratiquement insensible à $\Delta i^{p}$.

Notons qu'il est préférable de disposer également un autre filtre interférentiel $\left(\Phi_{3}\right.$, identique à $\left.\Phi_{2}\right)$ sur $F^{d}$ avant la cellule $\mathrm{C}_{1}$; ceci dans le but de perturber au minimum l'évolution du système atomique par la relaxation optique due à la composante $D$ qui n'est pas utilisée pour la détection.

On pourrait aussi utiliser pour $\mathrm{F}^{p}$ la première raie de résonance d'un alcalin, $\lambda_{1}$, et pour $F^{d}$ la deuxième raie de la série principale, $\lambda_{2}$ (par exemple pour $R b$, $\lambda_{1}=7800 \AA$ et $\lambda_{2}=4215 \AA$ ). Cependant, ceci suppose que l'on envisage une étude de relaxation pouvant se faire à forte tension de vapeur pour qu'une absorption optique appréciable soit mesurable sur $\lambda_{2}$. Lorsque la cellule $C_{1}$ contient un mélange d'alcalins (orientés l'un par pompage optique, l'autre par échange), une autre possibilité consiste à utiliser sur $\mathrm{F}^{v}$ et $\mathrm{F}^{d}$ les raies de résonance de chacun des deux éléments.

5) La méthode 2 présente enfin l'avantage de faire jouer à chacun des deux faisceaux $F^{p}$ et $F^{d}$ leur rôle propre, pompage ou détection :

- On peut ainsi faire un choix rationnel des caractéristiques spectrales et de polarisation appropriées à chacun d'eux.

- Il en résulte que l'on peut élargir le champ de 
recherche aux expériences pour lesquelles les caractéristiques nécessaires au pompage et à la détection ne sont pas réalisables sur un faisceau unique.

Pour illustrer ces points, nous envisageons deux exemples :

a) Choix des caractéristiques optiques des DeUX faisceaux $F^{p}$ et $F^{d}$. - Nous ne faisons, dans ce paragraphe, aucune hypothèse sur l'état initial dans lequel $\mathrm{F}^{p}$ doit préparer le système, ou sur l'observable $Q$ dont $\mathrm{F}^{d}$ doit suivre l'évolution. De manière générale, le profil spectral $u(k)$ des faisceaux lumineux $\mathrm{F}^{p}$ et $\mathrm{F}^{d}$ doit satisfaire aux conditions suivantes :

- $u\left(k_{0}\right)\left(k_{0}\right.$, fréquence de résonance atomique) doit être intense pour $\mathrm{F}^{v}\left(T_{p} \ll k_{i} T_{i}\right)$ pour que le pompage soit efficace et que le système soit préparé dans un état très différent de l'état de Boltzmann. Par contre $u\left(k_{0}^{\prime}\right)$ doit être peu intense pour $\mathrm{F}^{d}$ $\left(T_{d} \gg k_{i} T_{i}\right)$, pour ne pas introduire une relaxation optique indésirable.

- Sur $\mathrm{F}^{p}$ la largeur de $u(k)$ peut être quelconque : les fréquences $k$ qui ne sont pas réellement absorbées par la vapeur ne modifient pas l'état initial où se trouve le système après coupure de $\mathrm{F}^{p}$. Sur $\mathrm{F}^{d}$ il y $a$ avantage au contraire à réduire au maximum $u(k)$ pour $k \neq k_{0}^{\prime}$ : les fréquences $k$ qui ne sont pas absorbées par la vapeur ne participent pas à la détection du signal $L_{A}$ mais elles contribuent au bruit engendré par $\mathrm{F}^{d}$ au niveau de la photocathode de $\mathrm{PM}_{d}$.

Une lampe contenant l'alcalin et de l'argon (1 mm de mercure) excitée par une décharge H. F. émet une raie intense mais large (3 à 4 fois au moins la largeur Doppler). Elle convient bien pour $\mathrm{F}^{p}$, et mal pour $\mathrm{F}^{d}$. Par contre pour $\mathrm{F}^{d}$ une " lampe de résonance optique " qui émet une raie peu intense sur une largeur égale à la largeur Doppler est beaucoup mieux appropriée. C'est la raison pour laquelle nous utilisons sur $\mathrm{F}^{d}$ la lumière de résonance optique émise par la vapeur pure d'un alcalin sous faible tension de vapeur $\left(10^{-7}\right.$ à $10^{-6} \mathrm{~mm}$ de mercure); cette vapeur contenue dans la cellule $\mathrm{C}_{2}($ fig. 3 ) est excitée optiquement à angle droit de la direction de $\mathrm{F}^{d}$ par une lampe à décharge de type usuel, $\mathrm{S}_{2}$. Outre l'amélioration du rapport signal sur bruit, l'emploi d'une "lampe de résonance " présente d'autres avantages sur lesquels nous allons revenir.

b) Nouvelles expériences possibles. - Le fait que la méthode 1 impose une relation entre le procédé de pompage et la nature de l'observable détectée, s'est révélée particulièrement gênant pour généraliser aux divers alcalins l'étude de la relaxation de $<$ S.I $>$ [10]. Nous allons montrer que l'emploi de la méthode 2 permet d'étendre cette étude à d'autres alcalins que $R b$; nous en verrons l'intérêt au paragraphe III : cela permet la mesure des rapports entre les diverses sections efficaces d'échange.
Pour préparer le système atomique dans un état pour lequel on a $<\mathbf{S . I}>\neq 0$, on dispose de plusieurs procédés :

๙) Le premier (pompage hyperfin) consiste à exciter la vapeur par un faisceau de lumière naturelle ou polarisée linéairement (même probabilité d'absorption pour tous les sous-niveaux $\left|F m_{F^{\prime}}\right\rangle$ de même $F$ ) mais dont l'intensité $i_{2}^{p}$ de la composante hyperfine issue du niveau $F_{+}=I+1 / 2$ est différente de celle $i_{1}^{p}$ issue du niveau $F_{-}=I-1 / 2\left({ }^{1}\right)$.

Pour les intensités $i^{p}$ très fortes $\left(T_{p} \ll k_{H} T_{1 H}\right.$, $T_{1 H}$ étant le temps de relaxation de $<\mathbf{S}$.I $>$ défini par l'équation (8)), $\mathrm{F}^{p}$ permet de préparer le système dans un état initial $<\mathbf{S . I}>_{0}$ qui ne dépend pas de $i^{p}$ mais seulement de $\left(i_{2}^{p}-i_{1}^{p}\right) /\left(i_{2}^{p}+i_{1}^{p}\right)=\rho_{p}$. Notons que $\left|<\mathbf{S} . \mathbf{I}>_{0}\right|$ est une fonction croissante de $\left|\rho_{p}\right|$. En pratique, on utilise dans le cas de $\mathrm{Rb}$ les quasi-coïncidences existant entre les composantes hyperfines des deux isotopes ${ }^{85} \mathrm{Rb}$ et ${ }^{87} \mathrm{Rb}$ et l'on réalise ainsi facilement des valeurs de $\rho_{p}$ appréciables (de l'ordre de 0,5 ) c'est-à-dire un pompage hyperfin efficace $\left(\mid<\right.$ S.I $>_{0} \mid$ grand). Pour le Cs une technique analogue a été proposée [14] qui fait usage d'une lampe d'argon placée dans un champ magnétique de 5000 gauss : l'une des composantes Zeeman de la raie $8521,4 \AA$ de $A$ coïncide, à cette valeur du champ, avec l'une des composantes hyperfines de Cs. Cependant, il est difficile de faire émettre une telle source de manière intense et la réalisation du montage pose des difficultés non négligeables. Par suite, pour tous les alcalins autres que $\mathrm{Rb}$, on ne dispose, en pratique, que des sources usuelles intenses, qui émettent des raies très larges, plus ou moins renversées, et dont les composantes hyperfines ont des intensités comparables $\left(\rho_{p} \approx 0,1\right)$; le pompage hyperfin est inefficace et $\mid<$ S.I $>_{0} \mid$ petit.

$\beta)$ Nous avons utilisé une autre méthode qui consiste à exciter la vapeur dans la direction du champ $H_{0}$, par de la lumière polarisée circulairement. Même si l'on utilise une source usuelle pour laquelle $i_{2}^{p} \approx i_{1}^{p}$, on accroît considérablement la population du sous-niveau $F_{+}=I+1 / 2, m_{F}= \pm(I+1 / 2)$. On peut ainsi préparer le système atomique dans un état pour lequel $\langle\mathrm{S} . \mathrm{I}\rangle_{0}$ est grand et ceci quel que soit l'alcalin; mais en même temps, cet état possède une forte polarisation électronique $\left\langle S_{z}>_{0} \neq 0\right.$. Si l'on désire un état initial où $\langle\mathbf{S . I}\rangle_{0}$ est différent de zéro et où les polarisations électroniques longitudinales et transversales sont nulles, il suffit d'appliquer brutalement à la vapeur un champ de radiofréquence à la fréquence

(1) Nous supposons dans ce paragraphe que la structure hyperfine de l'état excité n'est pas résolue; il n'existe par suite que deux composantes hyperfines d'intensités $i_{1}$ et $i_{2}$ distantes de $\Delta W$, structure hyperfine de l'état fondamental. Nous envisageons en détail dans le paragraphe III les effets liés à l'existence d'une structure hyperfine résolue $\Delta ?{ }^{\circ}$ dans l'état excité (cas du césium). 
Zeeman $g_{F}^{\prime} \gamma_{S} H_{0}$ qui permet de détruire ces polarisations sans altérer $<\mathbf{S . I}\rangle_{\mathbf{0}}$ (nous reviendrons ci-après sur les précautions à prendre dans la réalisation de cette séquence).

On peut donc étudier la relaxation de $<$ S.I $>$ à partir de l'un ou l'autre des deux états précédents $\left(<S_{z}>_{0} \neq 0\right.$ et $\left\langle S_{z}>_{0}=0\right)$; nous avons vu qu'on s'attend théoriquement à ce que ces relaxations soient très différentes en présence de collisions d'échange entre atomes identiques (il y a couplage entre $<$ S.I $>$ et $\left\langle S_{z}\right\rangle$, cf. $\S$ I-3).

Pour détecter l'évolution de $<\mathbf{S . I}\rangle$ et étudier sa relaxation, les caractéristiques que l'on demande à $\mathrm{F}^{d}$ sont les suivantes :

- intensités différentes sur les deux composantes hyperfines : $i_{2}^{d} \neq i_{1}^{d}$;

- lumière naturelle ou polarisée linéairement.

Dans ces conditions, on montre [3] que la lumière absorbée sur $\mathrm{F}^{d}$ est donnée par l'expression suivante :

$$
L_{\mathrm{A}}=L_{0}+K<\mathbf{S} . \mathbf{I}>
$$

$L_{0}$ est la lumière absorbée par la vapeur à l'équilibre de Boltzmann ; $K$, et par suite la sensibilité de la détection, croît avec $\rho_{d}$ (analogue de $\rho_{p}$ ).

Lorsque $\mathrm{F}^{p}$ et $\mathrm{F}^{d}$ coïncident (méthode 1), seul le pompage hyperfin (procédé $\alpha$ ) est compatible avec les caractéristiques du faisceau lumineux nécessaires à la détection. Pour tous les alcalins autres que $\mathrm{Rb}, \rho_{d}=\rho_{p}$ est très petit : non seulement le pompage hyperfin est très mauvais, mais la détection elle-même est inefficace. Par contre, avec deux faisceaux $\mathrm{F}^{p}$ et $\mathrm{F}^{d}$ distincts (méthode 2), on peut utiliser l'autre type de pompage (procédé $\beta$ ) et obtenir ainsi de grandes valeurs de $<\mathbf{S . I}>_{0}$ qui sont détectables même si $\rho_{d}$ est faible. Mais on peut en outre augmenter $\rho_{d}$ par l'emploi sur $\mathrm{F}^{d}$ d'une lampe de résonance optique (cf. § III-1-c).

III. Application à l'étude de la relaxation de $<$ S.I $>$. - Nous avons appliqué la méthode 2, décrite ci-dessus, à l'étude de la relaxation de $<\mathbf{S . I}>$, en présence de collisions d'échange. Cela nous a permis, en particulier, d'obtenir la valeur des rapports des sections efficaces d'échange, Rb-Cs, Cs-Cs. L'extension des mesures aux autres alcalins est en cours. La réalisation expérimentale de la méthode a soulevé, dans le cas du Cs, des difficultés que nous examinons maintenant; nous décrirons ensuite les résultats obtenus.

1) Réalisation expérimentale. - Rappelons d'abord le dispositif. $\mathrm{F}^{p}$ dirigé suivant $H_{0}$ et polarisé en lumière circulaire, est filtré pour ne laisser passer que $\mathrm{D}_{2}\left({ }^{2}\right)$. Comme nous l'avons vu, on prépare

(2) Dans le cas du Cs nous réalisons des intensités pompantes beaucoup plus fortes avec $D_{2}$ qu'avec $D_{1}$ parce qu'une partie de l'optique de $\mathrm{F}^{p}$ est en plexiglas dont le coefficient d'absorption augmente très rapidement entre 8500 et $9000 \AA$. ainsi le système dans un état où $<$ S.I $>$ et $\left\langle S_{z}>\right.$ sont grands. Considérons le cas où nous nous intéressons à la relaxation de $\langle$ S.I $\rangle$ en absence de $<S_{z}>$.

La figure $4 a$ indique la valeur de l'intensité pompante $\mathrm{F}^{p}$, qui est coupée brusquement à l'instant 0 , (le temps de fermeture de l'obturateur, $T_{0}$, est de l'ordre de $\left.1,7 \times 10^{-3} \mathrm{~s}\right)$. A l'instant $T_{0}+\theta($ fig. $4 b)$, on applique à la vapeur le champ de radiofréquence $H_{1}$ (fréquence $\omega_{0}=g_{\mathrm{F}}^{\prime} \gamma_{\mathrm{s}} H_{0}$ ) qui permet de détruire $\left\langle S_{z}\right\rangle$ sans altérer $<$ S.I $>$. Les

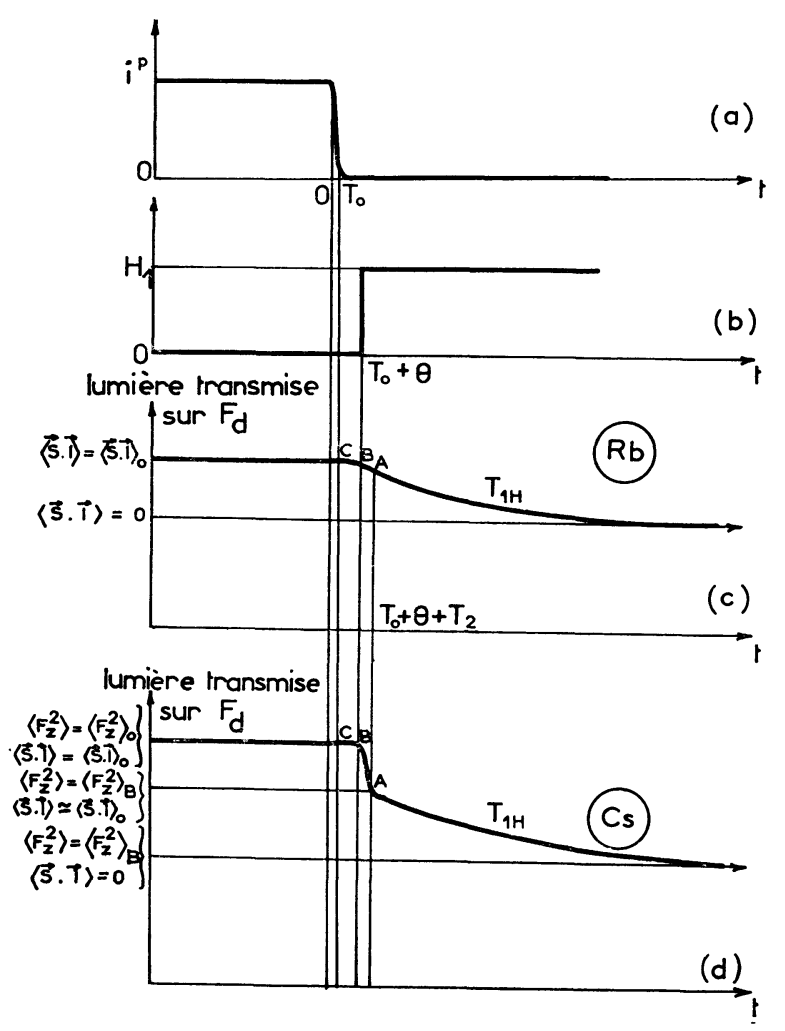

Fig. 4. - Séquence pour l'étude de la relaxation de $<$ S.I $>$ en l'absence de $\left\langle S_{z}>\right.$.

(a) Coupure de $\mathrm{F}^{p}$.

(b) Application de $H_{1}$.

(c) Transitoire observé sur la vapeur de $\mathrm{Rb}$.

(d) Transitoire observé sur la vapeur de Cs.

figures $4 c$ et $4 d$ représentent les transitoires observés sur le faisceau de détection $\mathrm{F}^{d}$ dans le cas de $\mathrm{Rb}$ et de Cs. Remarquons tout de suite qu'entre $\mathrm{T}_{0}$ et $T_{0}+\theta$, on observe la relaxation de $<$ S.I $>$ en présence de $\left\langle S_{z}\right\rangle$. Comme nous l'avons signalé au paragraphe I-3, elle est très longue si on la compare à celle de $<$ S.I $>$ en l'absence de $\left\langle S_{z}\right\rangle$. Ceci se traduit entre les points $\mathrm{C}$ et $\mathrm{B}$ des figures $4 c$ et $4 d$ par une brisure à peine détectable en $\mathrm{C}$, à l'instant $T_{0}$. [Notons que l'application de $H_{1}$ ne doit pas précéder l'instant $T_{0}$ ( $\theta$ doit être positif), sans quoi l'effet simultané du faisceau pompant et de $H_{1}$ 
provoquerait une annulation de $\langle$ S.I $\rangle$ en un temps de _l'ordre de $T_{p}$.]

a) Effet du champ $H_{1}$. - Nous examinerons maintenant de plus près l'effet du champ $H_{1}$, à partir de l'instant $T_{0}+\theta$. On montre que sous l'effet du champ $H_{1}$, le transitoire de $\left\langle P_{\mathrm{F}+} S_{z}\right\rangle$ et de $\left\langle P_{F_{-}} S_{z}\right\rangle$ est une sinusoïde de fréquence $g_{\mathrm{F}}^{\prime} \gamma_{\mathrm{S}} H_{1}$ exponentiellement amortie avec une constante de temps $T_{2}$; de même les aimantations transversales $\left\langle P_{\mathrm{F}+} S_{x}\right\rangle$ et $\left\langle P_{\mathrm{F}_{-}} S_{x}\right\rangle$ qui oscillent à la fréquence $\omega_{0}$ sont amorties avec la constante de temps $T_{2}$. Au bout du temps $T_{2}$, on doit donc observer la relaxation de $\langle\mathbf{S}$.I $\rangle$, caractérisée par la constante de temps $T_{1 H}$ (cf. éq. (8)) en l'absence de toute aimantation longitudinale ou transversale. $T_{2}$ dépend de la valeur du champ statique $H_{0}$ et de son inhomogénéité dans le volume de $C_{1}$. En agissant sur ces paramètres, on peut le rendre très court sans affecter $T_{1 H}$ en sorte que $T_{2} / T_{1 H} \ll 1$ (par exemple la mesure de $T_{1 H}$ a été faite sur Cs avec un rapport $T_{1 H} / T_{2}$ compris entre 5 et 15). Entre B et A (fig. $4 c, 4 d$ ) on devrait donc s'attendre à voir progressivement apparaître $T_{1 H}$, la mesure de cette constante de temps se faisant sur la partie du transitoire au-delà du point $A$. En réalité, l'expérience montre qu'entre $\mathrm{B}$ et $\mathrm{A}$ les transitoires relatifs au $\mathrm{Rb}(4 c)$ sont conformes à ce que nous venons de dire, alors qu'ils ont un aspect très différent pour le $\mathrm{Cs}(4 d)$. La détection de ce transitoire révèle ainsi la présence simultanée d'une autre observable que $\langle$ S.I $>$ dans le cas du Cs. Nous abordons ce point maintenant.

b) Signification physique de l'observable. - La signification physique de l'observable détectée sur $\mathrm{F}^{d}$ ne pose un problème que si la structure hyperfine $\Delta w$ de l'état excité n'est pas négligeable devant la largeur Doppler ; parmi tous les alcalins, ce cas ne se rencontre qu'avec Cs. La figure 5 représente les quatre composantes hyperfines de $D_{1}$ d'intensité $i_{2}^{\prime d}, i_{2}^{\prime \prime}, i_{1}^{\prime d}, i_{1}^{\prime \prime}$ qui parviennent au détecteur. Si l'on ne fait aucune hypothèse sur les valeurs

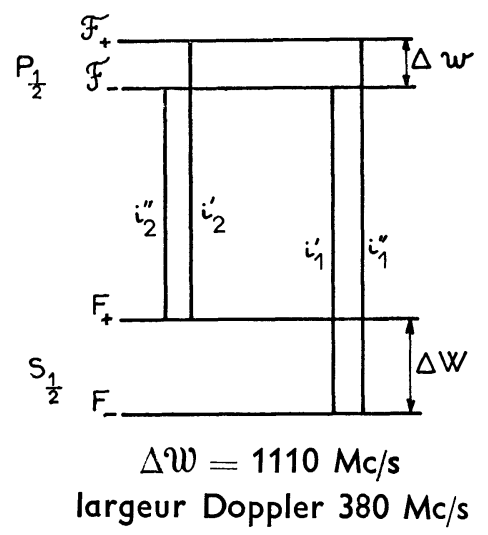

Fig. 5. - Structure hyperfine de la raie $D_{1}$. relatives de ces intensités mais si l'on suppose $\mathrm{F}^{d}$ composé de lumière $D_{1}$, naturelle, dirigée suivant $\mathrm{Ox}$ (conditions employées dans nos expériences sur le Cs), on obtient l'expression suivante de $L_{\mathrm{A}}$ (voir appendice) :

$$
\begin{aligned}
L_{\mathrm{A}} & =k\left(i_{1}^{\prime d}+i_{1}^{\prime \prime} d+i_{2}^{\prime d}+i_{2}^{\prime \prime} d\right) \\
& +k\left(i_{2}^{\prime d}+i_{2}^{\prime \prime}-i_{1}^{\prime d}-i_{1}^{\prime \prime}\right)<P_{F^{\prime}}-P_{F-}> \\
& +8 k\left(i_{2}^{\prime \prime} d-i_{2}^{\prime d}\right)<P_{F+}\left[g_{F}^{\prime}\left(F_{x}^{2}-\mathbf{F}^{2}\right)+\frac{1}{4}\right]> \\
& +8 k\left(i_{1}^{\prime \prime}-i_{1}^{\prime d}\right)<P_{F-}\left[g_{F}^{\prime 2}\left(F_{x}^{2}-\mathbf{F}^{2}\right)+\frac{1}{4}\right]>
\end{aligned}
$$

Le premier terme est indépendant de l'état du système atomique, le second est proportionnel à la différence de populations entre les deux niveaux hyperfins et permet de détecter son écart par rapport à l'équilibre de Boltzmann $\langle$ S.I $\rangle$, cf. éq. (2)) les troisième et quatrième termes font intervenir l'alignement dans $F_{+}$et dans $F_{-}$.

On peut montrer que l'expression (13) devient identique à (12) dans les deux cas suivants :

- l'excitation optique est telle que les conditions

$$
\begin{aligned}
& i_{2}^{\prime d}=i_{2}^{\prime \prime} d=i_{2}^{d} \\
& i_{1}^{\prime d}=i_{1}^{\prime \prime}=i_{1}^{d}
\end{aligned}
$$

soient simultanément satisfaites. C'est ce qui arrive en particulier pour tous les alcalins ayant une structure hyperfine $\Delta w$ non résolue $(\mathrm{Na}-\mathrm{K}-\mathrm{Rb})$;

- l'excitation optique est quelconque, mais l'alignement de la vapeur correspond à l'équilibre de Boltzmann

$$
<P_{\mathrm{F}_{ \pm}} F_{x}^{2}>_{B}=\frac{1}{3} F_{ \pm}\left(F_{ \pm}+1\right) .
$$

On peut montrer que, sous l'effet du champ de radiofréquence $H_{1}$, à résonance, le transitoire de $\left\langle F_{z}^{2}\right\rangle$ est une sinusoïde de fréquence $g_{\mathrm{F}}^{\prime} \gamma_{\mathrm{S}} H_{1}$ exponentiellement amortie avec la constante de temps $T_{2}$ vers la valeur $\left\langle F_{z}^{2}\right\rangle_{B} .\left\langle F_{x}^{2}\right\rangle$ évolue de manière plus complexe mais tend aussi exponentiellement vers $\left\langle F_{x}^{2}\right\rangle_{B}$ avec la constante de temps $T_{2}$. En conclusion, au bout du temps $T_{2}$, $L_{\mathrm{A}}$ ne dépend plus que de $\langle\mathbf{S . I}\rangle$ et permet l'étude de sa relaxation.

L'aspect de la séquence observée sur $\mathrm{F}^{d}$ dans le cas du Cs $(f i g .4 d)$ est conforme à ces prévisions : la variation rapide du signal optique entre B et A correspond à la variation de $\left\langle F_{x}^{2}\right\rangle$ sous l'effet de $H_{1}$ et à son retour vers la valeur d'équilibre de Boltzmann en un temps $T_{2}$. Le signal est exploité à partir du point $\mathrm{A}$ où une cassure apparaît.

La figure 6 donne un exemple du signal obtenu expérimentalement sur la vapeur de Cs à la température de $19^{\circ} \mathrm{C}$. 


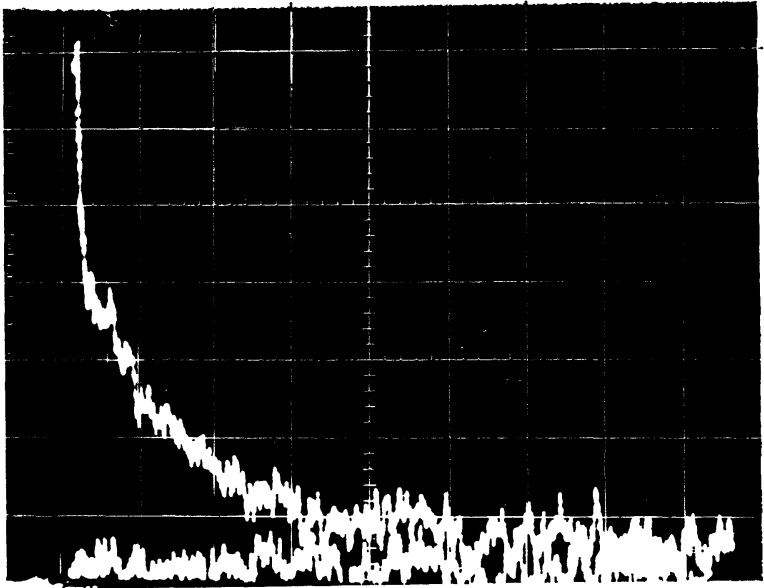

Fig. 6. - Variation du signal optique observé sur la vapeur de $\mathrm{Cs}$ à $19^{\circ} \mathrm{C}$. La variation rapide est due à la destruction de l'alignement lors de l'application de $H_{1}$ après coupure de $\mathrm{F}^{p}$. La variation lente représente la relaxation de $\left\langle\right.$ S.I $>$ en l'absence de $\left\langle S_{z}>\right.$.

Lorsqu'on opère non plus sur une vapeur pure mais sur un mélange d'alcalins, on est conduit pour les mesures de sections efficaces d'échange à détecter le signal dans des conditions où les temps de relaxation sont courts et où l'absorption optique est faible. Ceci nous conduit à envisager avec plus de détails les problèmes liés à la sensibilité de la détection.

c) Sensibilité de la détection. - Nous avons vu (§ II-5) que la sensibilité de la détection est d'autant meilleure que $\left|\rho_{d}\right|$ est plus grand et que le profil spectral de la raie détectrice est plus étroit.

Les deux composantes hyperfines sont émises par la vapeur dans le rapport des poids statistiques $I+1 / 2$ et $I-1 / 2$ des niveaux $F_{+}$et $F_{-}$; elles sont aussi réabsorbées dans ce rapport par une vapeur à l'équilibre de Boltzmann. Pour augmenter $\left|\rho_{d}\right|$, l'idée la plus simple consiste à filtrer la lumière qu'émet une source de Cs par de la vapeur de Cs. En fait ce procédé présente un grave inconvénient : pour être sélective, l'absorption par le filtre doit être très forte (d'autant plus que, pour $\mathrm{Cs}$, I est grand et le rapport $(I+1 / 2) /(I-1 / 2)$ voisin de 1). Comme l'absorption par le filtre est plus forte aux fréquences résonnantes que sur les ailes de la raie, elle a pour effet d'élargir considérablement le profil spectral. On peut remédier à cet inconvénient en utilisant pour filtre une vapeur présentant ellemême un grand écart de populations $\left\langle P_{F+}\right\rangle$ et $\left\langle P_{F-}\right\rangle$ par rapport à l'équilibre de Boltzmann : la réabsorption des composantes hyperfines devient ainsi très sélective.

En pratique, nous avons préféré ne pas utiliser de filtre, mais réaliser dans la source elle-même les conditions requises :

Nous utilisons sur $\mathrm{F}^{d}$ une " lampe de résonance 》 $\mathrm{C}_{2}$ (cf. fig. 3) afin que le profil spectral soit étroit ( $\left.{ }^{3}\right)$. Vers $30^{\circ} \mathrm{C}$ (pression de Cs environ de quelques $1^{-6} \mathrm{~mm}$ de mercure) l'expérience montre que la lumière de résonance émise par la cellule $C_{2}(6 \mathrm{~cm}$ de diamètre) fournit une intensité $i^{d}$ convenable et que la réabsorption dans $\mathrm{C}_{2}$ est suffisante pour que l'on obtienne $i_{1}^{d}>i_{2}^{d}$. Nous faisons du pompage optique sur la vapeur de $\mathrm{C}_{2}$ dans le but de rendre plus sélective la réabsorption des composantes hyperfines et d'améliorer $\left|\rho_{d}\right|$ : nous utilisons, comme lumière pompante, celle émise par $\mathrm{S}_{2}$ polarisée circulairement et se propageant dans le sens du champ magnétique où se trouve placée $\mathrm{C}_{2}$. La vapeur de $\mathrm{C}_{2}$ possède à la fois $\langle\mathbf{S} . \mathbf{I}\rangle \neq 0$ et $\left\langle S_{z}\right\rangle \neq 0$, par suite la lumière de résonance émise avec la polarisation $\pi$ ou $\sigma\left(\mathcal{L}^{\pi}\right.$ ou $\left.\mathcal{L}^{\sigma}\right)$ n'a pas le même profil spectral : $\rho_{d}$ n'est pas le même et les intensités $i_{2}^{\prime d}$, $i_{2}^{\prime d}\left(\right.$ ou $\left.i_{1}^{\prime d}, i_{1}^{\prime d}\right)$ des composantes hyperfines dues à la structure $\Delta w$ de l'état excité sont différentes. En outre, $\mathfrak{L}^{\pi}$ détecte $\left\langle F_{z}^{2}\right\rangle$ tandis que $\mathfrak{L}^{\sigma}$ détecte $\left\langle F_{y}^{2}\right\rangle$. Aussi les transitoires de relaxation utilisant soit $\mathcal{L}^{\pi}$, soit $\mathcal{L}^{\sigma}$ sont différents. La détection avec $\mathfrak{L}^{\sigma}$ se révèle plus intéressante pour l'étude de $\langle$ S.I $\rangle$ (cf. appendice).

En résumé, le signal obtenu par ce procédé de détection ( $f i g$. 6) représente un gain en sensibilité considérable par rapport à ce que permet d'obtenir une lampe usuelle de $C$ s placée sur $\mathrm{F}^{d}$. La figure 6 est relative à une séquence unique sur une vapeur de Cs pur. En pratique, nous faisons la moyenne des signaux obtenus au cours d'un grand nombre de séquences successives, à l'aide du "Enhancetron " (§ II-3). Ceci permet de détecter des variations de constantes de temps supérieures à $5 \%$, sur un mélange de $\mathrm{Rb}$ et Cs.

2) Résultats. - a) Étude de la relaxation de $<\mathbf{S} . \mathbf{I}\rangle$ en l'absence de $\left\langle S_{z}\right\rangle$ sur $R b, C s$, et sur un mélange Rb-Cs. - Nous pouvons ainsi mesurer les rapports des sections efficaces d'échange de spin $\mathrm{Rb}-\mathrm{Rb} / \mathrm{Rb}-\mathrm{Cs} / \mathrm{Cs}-\mathrm{Cs}$.

L'étude expérimentale a déjà été faite sur les deux isotopes de $\mathrm{Rb}\left({ }^{85} \mathrm{Rb},{ }^{87} \mathrm{Rb}\right.$ purs ou mélangés) [10] et sur des mélanges $\mathrm{Rb}-\mathrm{Cs}\left({ }^{85} \mathrm{Rb}-\mathrm{Cs}\right.$ et $\left.{ }^{87} \mathrm{Rb}-\mathrm{Cs}\right)$ [15] en employant la méthode 1 . Pour pouvoir déduire de ces mesures des valeurs absolues des sections efficaces d'échange, il faut connaître la tension de vapeur saturante de $\mathrm{Rb}$ et celle de $\mathrm{Cs}$ en fonction de la température. Pour le césium, plusieurs références fournissent des indications qui sont en bon accord. Par contre, il y a un facteur 3 d'écart entre les diverses valeurs relatives à $\mathrm{Rb}$. La dispersion des résultats de section efficace $\mathrm{Rb}-\mathrm{Rb}$ publiés tient dans certains cas à ce fait et le plus souvent à l'imprécision sur la nature de l'observable. La méthode

(3) Nous appelons désormais " lampe de résonance " la source constituée par une cellule contenant la vapeur pure d'un alcalin sous faible tension de vapeur et émettant la raie de résonance de l'élément. 
que nous allons décrire a l'avantage d'éliminer ces causes de dispersion; elle permet de mesurer directement le rapport entre deux sections efficaces différentes.

๙) Principe de mesure.

Il est possible de déterminer les proportions de $\mathrm{Rb}$ et de Cs en phase vapeur, en choisissant convenablement les proportions en phase solide [15].

- Nous avons trois cellules, la première (I) contenant du $\mathrm{Rb}$ pur $\left(N_{\mathrm{Rb}}\right.$ atomes $\left.\mathrm{de} \mathrm{Rb} / \mathrm{cm}^{3}\right)$, la seconde (II) du Rb et du Cs $\left(n_{\mathrm{Rb}}\right.$ et $n_{\mathrm{Cs}}$ atomes de $\mathrm{Rb}$ et $\mathrm{Cs}$ par $\left.\mathrm{cm}^{3}\right)$, la troisième (III) du Cs pur $\left(N_{\mathrm{Cs}}\right.$ atomes de césium par $\mathrm{cm}^{3}$ ). Le rapport $n_{\mathrm{Rb}} / N_{\mathrm{Rb}}$ a été mesuré par comparaison de l'absorption optique de la raie de résonance $\mathrm{du} R \mathrm{Rb}$ par (I) et (II) ; il a été fait de même pour $n_{\mathrm{Cs}} / N_{\mathrm{Cs}}$.

- Nous mesurons la relaxation de $<$ S.I $>_{\mathrm{Rb}}$ sur (I) et (II) et celle de $<\mathbf{S . I}\rangle_{\text {Cs }}$ sur (II) et (III). Nous avons, après soustraction de la relaxation due à la paroi (sa contribution s'obtient en extrapolant à zéro les tensions de vapeur des alcalins) :

dans (I)

$$
\begin{aligned}
\frac{\mathrm{d}}{\mathrm{d} t}< & \mathbf{S} . \mathbf{I}>_{\mathrm{kb}} \\
& =-A(\mathrm{Rb}, \mathrm{Rb}) \sigma_{\mathrm{Rb}-\mathrm{Rb}}^{2} N_{\mathrm{Rb}}<\mathrm{S} . \mathrm{I}>_{\mathrm{Rb}}
\end{aligned}
$$

dans (II)

$$
\left\{\begin{array}{r}
\frac{\mathrm{d}}{\mathrm{d} t}<\mathrm{S} . \mathrm{I}>_{\mathrm{Rb}}=-\left[A(\mathrm{Rb}, \mathrm{Rb}) \sigma_{\mathrm{Rb}-\mathrm{Rb}}^{2} n_{\mathrm{Rb}}\right. \\
\left.+A(\mathrm{Rb}, \mathrm{Cs}) \sigma_{\mathrm{Rb}-\mathrm{Cs}}^{2} n_{\mathrm{Cs}}\right]<\mathrm{S} . \mathrm{I}>_{\mathrm{Rb}} \\
\frac{\mathrm{d}}{\mathrm{d} t}<\mathrm{S} . \mathbf{I}>_{\mathrm{Cs}}=-\left[A(\mathrm{Rb}, \mathrm{Cs}) \sigma_{\mathrm{Rb}-\mathrm{Cs}}^{2} n_{\mathrm{Rb}}\right. \\
\left.+A(\mathrm{Cs}, \mathrm{Cs}) \sigma_{\mathrm{Cs}-\mathrm{Cs}}^{2} n_{\mathrm{Cs}}\right]<\mathrm{S} . \mathbf{I}>_{\mathrm{Cs}}
\end{array}\right.
$$

$\operatorname{dans}($ III)

$\frac{\mathrm{d}}{\mathrm{d} t}<\mathbf{S} . \mathbf{I}>_{\mathrm{Cs}}=-A(\mathrm{Cs}, \mathrm{Cs}) \sigma_{\mathrm{Cs}-\mathrm{Cs}}^{2} N_{\mathrm{Cs}}<\mathbf{S} . \mathbf{I}>_{\mathrm{Cs}}$

expression où $A(\alpha, \beta)=2 \sqrt{2 \pi R T\left(\frac{1}{M_{\alpha}}+\frac{1}{M_{\beta}}\right)}$.

Puisque nous connaissons $n_{\mathrm{Rb}} / N_{\mathrm{Rb}}$ et $n_{\mathrm{Cs}} / N_{\mathrm{Cs}}$, de (15) et (16), nous tirons $A(\mathrm{Rb}, \mathrm{Cs}) \sigma_{\mathrm{Rb}-\mathrm{Cs}}^{2} n_{\mathrm{Cs}}$ qui, comparé à $(18)$, nous donne $\sigma_{\mathrm{Cs}-\mathrm{Cs}}^{2} / \sigma_{\mathrm{Rb}-\mathrm{Cs}}^{2}$; de même, nous obtenons $\sigma_{\mathbf{R b}-\mathbf{R b}}^{2} / \sigma_{\mathbf{R b}-\mathrm{Cs}}^{2}$ à l'aide de (15), (17) et (18) et nous en déduisons $\sigma_{\mathrm{Rb}-\mathrm{Rb}}^{2} / \sigma_{\mathrm{Cs}-\mathrm{Cs}}^{2}$ sans avoir besoin des tension de vapeur saturante de $\mathrm{Cs}$ et $\mathrm{de} \mathrm{Rb}$.

Sur l'équation (16), nous voyons que l'erreur relative sur $A(\mathrm{Rb}, \mathrm{Cs}) \sigma_{\mathrm{Rb}}^{2}-\mathrm{Cs} n_{\mathrm{Cs}}$ est d'autant plus faible que ce terme est plus grand : pour déterminer $\sigma_{\mathrm{Cs}-\mathrm{Cs}}^{2} / \sigma_{\mathrm{Rb}-\mathrm{Cs}}^{2}$, il y a avantage à ce que la relaxation de $\mathrm{Rb}$ soit dominée par l'effet des collisions sur le Cs. Il faut cependant qu'il y ait suffi- samment de $\mathrm{Rb}$ sinon les signaux seraient trop petits pour connaître de façon précise $\frac{\mathrm{d}}{\mathrm{d} t}<\mathbf{S} . \mathbf{I}>_{\mathrm{Rb}}$; mais l'utilisation du "Enhancetron " permet de faire des mesures sur des cellules contenant une proportion d'atomes de $\mathrm{Rb}$ très faible. Notons que par ailleurs, l'erreur relative sur $n_{\mathrm{Rb}} / N_{\mathrm{Rb}}$ et $n_{\mathrm{Cs}} / N_{\mathrm{Cs}}$ dépend peu de la valeur de ces rapports. On voit donc que pour déterminer $\sigma_{R b-R b}^{2} / \gamma_{R b-C s}^{2}$ de manière précise, il faut une cellule II contenant plus de Cs que de $\mathrm{Rb}$. On voit de même (éq. (17)) que pour déterminer $\sigma_{\mathrm{Cs}-\mathrm{Cs}}^{2} / \sigma_{\mathrm{R} b-\mathrm{Cs}}^{2}$ il faut une cellule II contenant plus de $\mathrm{Rb}$ que de Cs.

ß) Résultats.

Sur la cellule (I), nous avons vérifié que les résultats obtenus par la méthode 2 étaient en très bon accord avec les résultats antérieurs.

Jusqu'ici, les mesures ont été effectuées sur des cellules (II) contenant plus de césium que de rubidium.

La première, contenant $\mathrm{du}{ }^{85} \mathrm{Rb}$ et $\mathrm{du} \mathrm{Cs}$, avec $\left(n_{\mathrm{as} \mathrm{Rb}} / N_{\mathrm{sb}_{\mathrm{Rb}}}\right)=0,51$ et $\left(n_{\mathrm{Cs}} / N_{\mathrm{Cs}}\right)=0,46$, nous a donné $\left(\sigma_{\mathrm{Cs}}^{2}-\mathrm{Cs} / \sigma_{\mathrm{sb}}^{2}{ }_{\mathrm{Rb}}\right)=1,01$.

La seconde, contenant du ${ }^{87} \mathrm{Rb}$ et du $\mathrm{Cs}$, avec $\left(n_{{ }^{\mathrm{r}} \mathrm{Rb}} / N_{{ }^{{ }^{\mathrm{Rb}}}}\right)=0,21$ et $\left(n_{\mathrm{Cs}} / N_{\mathrm{Cs}}\right)=0,84$, nous a donné $\left(\sigma_{\mathrm{Cs}}^{2}-\mathrm{Cs}_{\mathrm{s}} / \sigma_{\mathrm{r}}^{2}{ }_{\mathrm{Rb}-\mathrm{Cs}}\right)=1,08$.

Une méthode analogue, sur une cellule contenant une forte proportion de césium (environ $85 \%$ du nombre total d'atomes) et une faible proportion de ${ }^{85} \mathrm{Rb}$ et de ${ }^{87} \mathrm{Rb}$ (environ $7 \%$ de chaque) a permis de montrer que $\sigma_{\mathrm{s}_{\mathrm{Rb}}}^{2} \mathrm{Cs}=\sigma_{\mathrm{s}_{\mathrm{Rb}}-\mathrm{Cs}}^{2}$ à $3 \%$ près.

Par suite à la précision des mesures

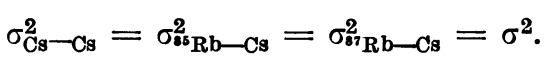

Par des mesures d'absorption optique, nous avons comparé les tensions de vapeur de Cs des cellules II et III à celle d'une cellule de Cs pur volontairement contaminée. Si l'on évalue la tension de vapeur dans cette dernière à l'aide de la formule donnée par Taylor et Langmuir [16] on obtient pour valeur absolue $\pi \sigma^{2}=2,4 \times 1^{-14} \mathrm{~cm}^{2}$. Notons que Moos et Sands ont mesuré $\pi \sigma_{\text {Cs }}^{2} \longrightarrow$ cs par une méthode différente [17] ; en prenant une définition de la section efficace identique à la nôtre, leur résultat conduit à $\pi \sigma^{2}=2,6 \times 10^{-14} \mathrm{~cm}^{2}$. L'accord avec le résultat ci-dessus paraît donc excellent.

Des mesures sur une cellule II, contenant peu de cé ium et beaucoup de rubidium sont en cours ; elles doivent permettre d'obtenir la valeur du rapport $\sigma_{\mathbf{R b}-\mathbf{R b}}^{2} / \sigma_{\mathbf{R b}}^{2}-\mathrm{Cs}$.

b) Étude de la relaxation de $<\mathbf{S}$.I $>$ en présence de $\left\langle S_{z}>\operatorname{sur} R b\right.$. - Nous avons indiqué au paragraphe III-1 comment procéder pour étudier la relaxation de $<\mathbf{S}$.I $>$ en l'absence et en présence de $\left\langle S_{z}>\right.$. La variation du signal optique tel qu'il est enregistré dans chacun de ces deux cas est reproduite ( $f g .7$ ) pour une vapeur de ${ }^{85} \mathrm{Rb}$ à $25{ }^{\circ} \mathrm{C}$ $\left(T_{\in H} \approx 0,1 \mathrm{~s}\right)$ contenue dans une cellule enduite de 


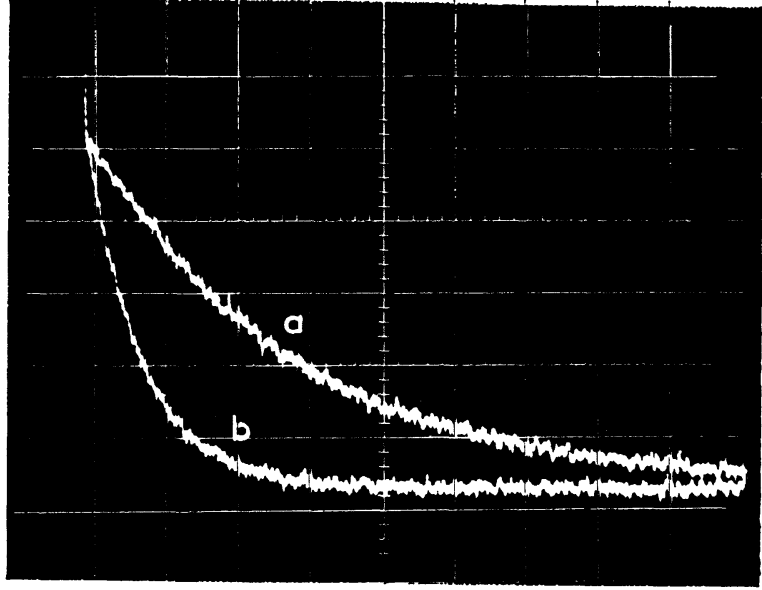

FIG. 7. - Variation du signal optique observé sur la vapeur de ${ }^{85} \mathrm{Rb}$ à $25^{\circ} \mathrm{C}$.

a) Relaxation de $\langle$ S.I $>$ en présence de $\left\langle S_{z}\right\rangle(\tau)$.

b) Relaxation de $\langle$ S.I $\rangle$ en l'absence de $<S_{z}>\left(T_{1}\right.$ H $)$.

paraffine deutérée $\left(T_{\mathrm{e}}=0,4 \quad \mathrm{~s}, \quad T_{n}=1,8 \mathrm{~s}\right.$, $\left.T_{H}=0,5 \mathrm{~s}\right)$. On constate que la relaxation de $\left\langle\mathbf{S . I}>\right.$ en présence de $\left\langle S_{z}>\right.$ (courbe $a$ ) est caractérisée par une constante de temps $\tau$ tout à fait comparable aux temps de relaxation dus au seul effet des collisions sur la paroi ; elle est très lente devant la relaxation de $\langle\mathbf{S}$.I $\rangle$ en l'absence de $\left\langle S_{z}\right\rangle$ (courbe $b$ ) caractérisée par la constante de temps $T_{1 H}$ qui fait intervenir les collisions d'échange $\left(T_{1 H} \simeq T_{\mathrm{e} H}\right)$. Ces résultats s'accordent tout à fait avec les prévisions théoriques énoncées au paragraphe I-3.

Nous avons observé une variation de la "pseudo" constante de temps $\tau$ avec la valeur initiale de $\left\langle\right.$ S.I $>_{0}$ (en diminuant $i^{p}$ on fait décroître $\left\langle S_{z}\right\rangle_{0}$ et par suite $\left.\left.<\mathbf{S . I}\right\rangle_{0}\right)$. Nous avons constaté que $\tau$ s'allonge de $20 \%$ quand $<$ S.I $>_{0}$ décroît par un facteur 10 ; $\tau$ est toujours compris entre $T$ et $T^{\prime}$ et très voisin de $T^{\prime}$ pour les polarisations initiales faibles (comme prévu au $§ \mathrm{I}-3$ ).

IV. Application à l'étude de la relaxation de $<S_{z}>$. - Nous avons appliqué la méthode 2 à l'étude de la relaxation de $\left\langle S_{z}\right\rangle$ en vue d'étudier l'effet des collisions d'un alcalin sur un gaz étranger. Une telle étude nécessite un grand nombre de mesures (à différentes pressions du gaz étranger) et l'avantage de la rapidité de la méthode 2 est, entre autres, particulièrement appréciable. Les variantes par rapport au montage précédent sont mineures. $\mathrm{F}^{p}$, dirigé suivant $\mathbf{H}_{0}$, filtré de manière à ne laisser passer que $D_{1}$, est polarisé circulairement. $F^{d}$ fait l'angle $\alpha=45^{\circ}$ avec $\mathbf{H}_{0}$, est filtré de manière à ne laisser passer que $D_{2}$ et traverse soit un polariseur circulaire situé avant $\mathrm{C}_{1}$, soit un analyseur circulaire situé après $\mathrm{C}_{1}$.

Nous avons choisi $D_{1}$ pour le pompage parce qu'ainsi le signe de la polarisation $<S_{z}>_{0}$ obtenue est le même quelle que soit la pression $p$ du gaz étranger et sa valeur absolue dépend peu de $p$ si $i^{p}$ est suffisamment grand (dans le pompage avec $D_{2}$ il existe pour tous les gaz une valeur de $p$ pour laquelle $<S_{z}>_{0}=0$, et cela quel que soit $i^{p}$ : le signe de $<S_{z}>_{0}$ change lorsqu' on passe du pompage "type Kastler " au pompage " type Dehmelt " [2]. De plus, aux très fortes pressions de gaz, le pompage Dehmelt aboutit à une polarisation beaucoup plus faible avec $D_{2}$ qu'avec $D_{1}$ ).

Notons aussi que nous aurions pu adopter un montage où les faisceaux $\mathrm{F}^{p}$ et $\mathrm{F}^{d}$ ont des directions symétriquement inclinées par rapport à $H_{0}$; nous avons rejeté cette solution parce qu'elle présente l'inconvénient de réduire $<S_{z}>_{0}$ pour une valeur donnée de $i^{p}$.

Au cours de la mise au point du procédé de détection, nous avons rencontri, des problèmes liés à la signification physique de l'observable et à la sensibilité de la détection.

1) Signification PhysiQue de L'obsertable. Le signal mesuré sur la lumière absorbée de $F^{d}$ se calcule directement à partir de la référence [3]. En utilisant les mêmes notations, nous obtenons, pour une détection par $D_{2}$ avec une polarisation

$$
\mathbf{e}_{\lambda} \pm=1 / \sqrt{2}\left( \pm i u_{x}+u_{y} \cos \alpha+u_{z} \sin \alpha\right)
$$

et une largeur de raie grande devant $\Delta w$ :

$$
\begin{aligned}
L_{\mathrm{A}}^{ \pm}=4 k\left(i_{1}^{d}+i_{2}^{d}\right) & <1 \pm S_{z} \cos \alpha> \\
+4 k\left(i_{2}^{d}-i_{1}^{d}\right) & <P_{F^{\prime}}\left(1 \pm S_{z} \cos \alpha\right) \\
& -P_{F-}\left(1 \pm S_{z} \cos \alpha\right)>
\end{aligned}
$$

Les deux indices \pm de $L_{\mathrm{A}}$ caractérisent chacun des deux états de polarisation circulaire repérés par continuité à partir des états $\sigma^{+}$ou $\sigma^{-}$correspondant à $\alpha=0(\mathbf{4})$. Si la condition

$$
i_{1}^{d}=i_{2}^{d}
$$

est réalisée, $L_{\mathrm{A}}$ dépend linéairement de $\left\langle S_{z}\right\rangle$ et permet l'étude de sa relaxation. Sinon, on voit que l'interprétation de $L_{\mathrm{A}}$ est délicate : elle fait intervenir aussi $\left\langle I_{z}\right\rangle$ et $<$ S.I $>$. Comme nous l'avons vu, $<I_{z}>$ n'est pas gênant quand il s'agit de mesurer les constantes de temps $T_{\mathrm{e}}$ et $T_{n}$ (§ I-1). Par contre, la présence de $\langle\mathbf{S}$.I $>$ dans le signal

(4) L'expression (19) tient compte de ce que la valeur moyenne de l'aimantation transversale est nulle dans le type d'expérience que nous envisageons. S'il n'en était pas ainsi, $L_{\mathrm{A}}$ s'exprimerait en fonction de l'observable $S_{z} \cos \alpha+S_{y} \sin \alpha$ au lieu de $S_{z} \cos \alpha$ seul. 
optique détecté est très gênante : nous avons vu que l'évolution de $\langle$ S.I $\rangle$ en présence de $\left\langle S_{z}\right\rangle$ se fait avec la pseudo constante de temps $\tau$ (§ I-3).

Expérimentalement, nous pousons déterminer si la condition (20) est satisfaite. En effet, moyennant (20), les signaux optiques détectés au cours de la relaxation sur $L_{\mathbf{A}}^{+}$ou $L_{\mathbf{A}}^{-}$sont symétriques l'un de l'autre $\left(\Delta L_{\mathbf{A}}^{+}=-\Delta L_{\mathbf{A}}^{-}\right)$et aucun signal n'est détecté sur la lumière absorbée sur $\mathrm{F}^{d}$ non polarisé $\left(\Delta\left(L_{\mathbf{A}}^{+}+L_{\mathbf{A}}^{-}\right)\right.$ est $n u l)$.

Si les signaux optiques $\Delta L_{\mathrm{A}}^{+}$et $\Delta L_{\mathrm{A}}^{-}$ne sont pas symétriques (ou si le signal $\Delta\left(L_{\mathbf{A}}^{+}+L_{\mathbf{A}}^{-}\right)$n'est pas nul), on peut en conclure que la condition (20) n'est pas réalisée. Ce test permet facilement de déceler une faible différence entre $i_{1}^{d}$ et $i_{2}^{d}$ et d'évaluer $\rho_{d}$ : la mesure de $\Delta L_{\mathbf{A}}^{+}, \Delta L_{\mathbf{A}}^{-}, \Delta\left(L_{\mathbf{A}}^{+}+L_{\mathbf{A}}^{-}\right)$fournit le rapport $A$ entre les amplitudes des signaux optiques représentant respectivement l'évolution de $\langle$ S.I $>$ et celle de $\left\langle S_{z}\right\rangle$. Pour les petites valeurs de $\left|\rho_{d}\right|$ on obtient :

$$
\begin{aligned}
A=\frac{2 \Delta\left(L_{\mathbf{A}}^{+}+L_{\mathbf{A}}^{-}\right)}{\Delta L_{\mathbf{A}}^{+}-\Delta L_{\mathbf{A}}^{-}} \\
=\frac{\left(i_{d}^{1}-i_{d}^{2}\right) \times 4 g_{F}^{\prime}<\mathbf{S} . \mathbf{I}>_{0}}{\left(i_{d}^{1}+i_{d}^{2}\right) \cos \alpha<S_{z}>_{0}} .
\end{aligned}
$$

De cette relation on déduit $\rho_{d}$ connaissant dan; l'état initial $<\mathbf{S} . \mathbf{I}\rangle_{0}$ et $\left\langle S_{z}\right\rangle_{0}$. Par exemple une asymétrie de $20 \%$ entre $\Delta L_{\mathrm{A}}^{+}$et $\Delta L_{\mathrm{A}}^{-}(A=0,2)$ correspond à une valeur de $\rho_{d}$ seulement de l'ordre de $7 \times 10^{-2}$ (pour $\alpha=45^{\circ}$ et un état initial très orienté : $\left\langle S_{z}>_{0} \simeq 1 / 2,4 g_{F}^{\prime}<\mathbf{S} \mathbf{I}>_{0} \simeq 1\right.$ ).

Expérimentalement, nous avons touịours observé, même avec la lumière de résonance de $R b$, émise par $\mathrm{C}_{2}$ (non pompé optiquement), une certaine asymétrie entre $\Delta L_{\mathbf{A}}^{+}$et $\Delta L_{\mathbf{A}}^{-}$signifiant une différence entre $i_{1}^{d}$ et $i_{2}^{d}$ dans le sens $\rho_{d}<0$, ceci corres. pond à une source trop renversée. On observe aussi que $\left|\rho_{d}\right|$ est supérieur pour une détection avec $D_{2}$ qu'avec $\mathrm{D}_{1}$ (ceci s'explique parce que la réabsorption est deux fois plus importante pour $D_{2}$ ). Notons d'autre part l'influence du facteur $\cos \alpha$. pour une valeur de $\rho_{d}$ fixée, l'asymétrie est d'autant plus grande que $\mathrm{F}^{d}$ est plus incliné par rapport à $H_{n}$.

En conséquence, avec les caractéristiques choisies pour $\mathrm{F}^{d}\left(\alpha=45^{\circ}\right.$ - lumière $\left.\mathrm{D}_{2}\right)$ et les sources dont nous disposons (y compris les "lampes de résonance "), nous avons dû renoncer à détecter $\left\langle S_{z}\right\rangle$ sur $L_{\mathbf{A}}^{+}$ou $L_{\mathbf{A}}^{-}$(cependant, ces mêmes sources avaient permis de détecter $\left\langle S_{z}\right\rangle$ avec une bonne apprc ximation par la méthode 1 parce que les caractéristiques de la détection étaient différentes : $\alpha=0$ - lumière $D_{1}$ ).

Le problème, qui consiste à modifier les caractéristiques d'une source de manière à obtenir que pour $D_{2}\left|\rho_{d}\right| \simeq 0$, s'est révélé très long et délicat à résoudre. Nous avons préféré adopter la solution suivante. Au lieu d'observer $L_{\mathbf{A}}^{+}$ou $L_{\mathbf{A}}^{-}$nous obser- vons la différence : $L_{\mathbf{A}}^{+}-L_{\mathbf{A}}^{-}$. A partir de (19), on obtient

$$
\begin{aligned}
L_{\mathbf{A}}^{-}-L_{\mathbf{A}}^{+}= & 8 k \cos \alpha\left[\left(i_{1}^{d}+i_{2}^{d}\right)<S_{z}>\right. \\
& \left.\left.+\frac{i_{2}^{d}-i_{1}^{d}}{2 I+1}\left(<S_{z}>+<I_{z}\right\rangle\right)\right]
\end{aligned}
$$

$L_{\mathbf{A}}^{+}-L_{\mathbf{A}}^{-}$ne fait pas intervenir l'observable $\langle\mathbf{S . I}\rangle$ mais seulement $\left\langle S_{z}\right\rangle$ et une faible proportion $\rho_{d} I(2 I+1)$ de l'observable $\left\langle I_{z}\right\rangle$; ce signal nous permet de mesurer $T_{\mathrm{e}}$ et $T_{n}$.

Nous avons représenté sur la figure 8 les modifications expérimentales apportées au dispositif de

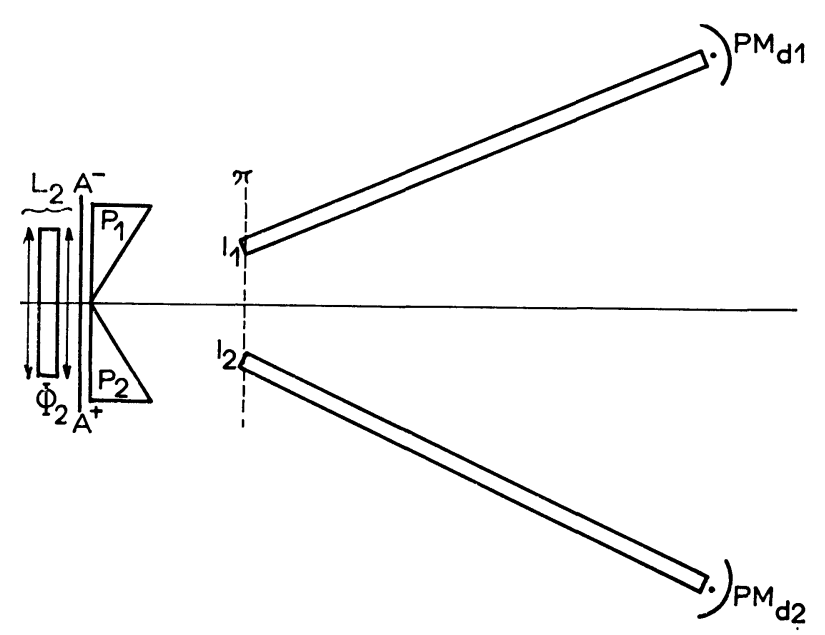

FIG. 8. - Montage optique utilisé sur le faisceau détecteur pour observer $L_{\mathbf{A}}^{-}-L_{\mathbf{A}}^{+}$et mesurer les constantes de temps de relaxation associées à $\left\langle S_{z}\right\rangle$.

la figure 3 pour permettre l'observation de $L_{\mathrm{A}}^{-}-L_{\mathrm{A}}^{+}$. Aux lentilles $L_{2}$ sont accolés deux prismes identiques $\mathrm{P}_{1}, \mathrm{P}_{2}$ dont une arête commune passe par le centre optique de $L_{2}$. Le faisceau $F^{d}$ est ainsi dédoublé en deux moitiés déviées dans deux directions symétriques par rapport à l'axe ; chaque moitié traverse entre $L_{2}$ et $P_{1}$ (ou $P_{2}$ ) un analyseur circulaire $A_{1}$ (ou $A_{2}$ ) droit (ou gauche). Dans le plan $\pi$ conjugué de $L_{1}$ par rapport à $L_{2}$, on obtient deux (pseudo) images $I_{1}$ et $I_{2}$. On mesure leur intensité à l'aide des photomultiplicateurs (Dumont K 1430) $\mathrm{PM}_{d 1}$ et $\mathrm{PM}_{d 2}$ précédés d'une canne de plexiglas poli jouant l'office de fontaine lumineuse $(\varnothing 20 \mathrm{~mm})$. Les courants photoélectriques sont directement appliqués aux deux entrées de l'amplificateur différentiel continu de l'oscilloscope Tektronix $502 \mathrm{~A}$. On observe ainsi sur l'écran l'évolution de $L_{\mathbf{A}}^{-}-L_{\mathbf{A}}^{+}$. Quelques réglages sont nécessaires pour équilibrer ce montage en pont : réglage d'optique pour que l'éclairement des images $I_{1}$ et $I_{2}$ soit le même, réglage de la tension appliquée à $\mathrm{PM}_{d 1}$ et $\mathrm{PM}_{d 2}$ pour que leur sensibilité soit la même. 
Ce montage a en même temps l'avantage d'éliminer les fluctuations du signal dues aux instabilités de la source : chaque point de la source éclaire uniformément $L_{1}$; une fluctuation d'intensité en un point doit donc engendrer la même fluctuation sur les photocathodes de $\mathrm{PM}_{d 1}$ et $\mathrm{PM}_{d 2}$ uniformément répartie sur chaque photocathode et par suite la même fluctuation de courant sur les signaux $L_{\overline{\mathbf{A}}}^{-}$ et $L_{\mathrm{A}}^{+}$: elle s'élimine sur la différence.

En pratique, nous utilisons comme source une “ lampe de résonance " (dispositif $\mathrm{S}_{2}, \mathrm{C}_{2}$ dessiné fig. 3). Parmi toutes les sources que nous avons essayées, c'est en effet celle qui fournit la valeur de $\left|\rho_{a}\right|$ la plus faible à condition que la tension de vapeur de $\mathrm{C}_{2}$ soit faible (quelques $10^{-7} \mathrm{~mm}$ ).

2) Sensibilité de la détection. - Grâce à la mise au point du montage en pont que nous venons de décrire, il est possible de mesurer $T_{\mathrm{e}}$ et $T_{n}$ sur des courbes de relaxation qui présentent un rapport signal sur bruit de l'ordre de 30 pour le $\mathrm{Rb}$ à la température de la pièce. Nous avons vérifié, sur $\mathrm{Rb}$, que par cette méthode, nous retrouvons les constantes de temps de relaxation mesurées par la méthode 1 sur des cellules sans gaz étranger enduites de paraffine [5] ainsi que sur des cellules contenant du néon à une pression de plusieurs $\mathrm{cm}$ de $\mathrm{Hg}$.

Conclusion. - Les méthodes d'étude de relaxation que nous avons décrites sont susceptibles d'assez nombreuses applications : elles peuvent s'appliquer à divers atomes alcalins, à divers problèmes de relaxation (par échange, par collisions sur la paroi ou contre un gaz, par excitation optique), à diverses observables. En particulier, ces méthodes nous ont permis de comparer les sections efficaces d'échange de spin ${ }^{85} \mathrm{Rb}-\mathrm{Cs},{ }^{87} \mathrm{Rb}$-Cs et $\mathrm{Cs}-\mathrm{Cs}$ et de montrer qu'elles sont égales à la précision des déterminations expérimentales.

De plus le fait de séparer les deux rôles du faisceau lumineux nous a permis à la fois d'améliorer les conditions de pompage et de détection. Ces améliorations présentent un intérêt non seulement pour les études de relaxation, mais pour une catégorie plus vaste de problèmes ayant trait au pompage optique sur les alcalins.

Nous tenons à remercier M. J. Brossel de l'aide qu'il nous a apportée au cours de la réalisation de ce travail.

\section{APPENDICE}

Nous partons des équations (1) et (2) de la référence [3]

$$
L_{\Lambda}\left(\mathbf{e}_{\lambda_{\mathbf{0}}}\right)=\sum_{\substack{F m_{F} \\ F^{\prime} m_{F^{\prime}}^{\prime}}} C_{F^{\prime} m_{F^{\prime}}^{F}}^{F m_{F}} \sigma_{F m_{F}^{\prime}}^{F^{\prime} m_{F^{\prime}}^{\prime}}
$$

$$
C_{\mathscr{F}^{m^{\prime} F^{\prime}}}^{F m_{F^{\prime}}}=\sum_{\mathscr{F} m_{\mathscr{F}}} \frac{1}{2}\left(\Gamma_{F \mathscr{F}}^{\prime}+\Gamma_{F^{\prime} \mathfrak{F}^{\prime}}^{\prime}\right)
$$

$<F m_{F}\left|\mathbf{e}_{\lambda_{0}} \cdot \mathbf{D}\right| \mathscr{F} m_{\mathscr{F}}><\mathscr{F} m_{\mathscr{F}}\left|\mathbf{e}_{\lambda_{0}}^{*} \cdot \mathbf{D}\right| F^{\prime} m_{F^{\prime}}^{\prime}>$. (2)

Nous faisons les hypothèses suivantes : excitation par $\mathrm{D}_{1}$ en l'absence de cohérences hyperfines.

Nous posons $\mathscr{F}=F, \mathcal{F}^{\prime}=F^{\prime}$ et $F^{\prime} \neq F$

$$
\begin{aligned}
& C_{F m_{F}^{\prime}}^{F m_{F}}=\Gamma_{F \mathscr{F}}^{\prime} \sum_{m_{\mathcal{F} F}}<F m_{r^{\prime}}\left|\boldsymbol{\epsilon}_{\lambda_{0}} . \mathbf{D}\right| \mathcal{F} m_{\mathscr{F}}> \\
& <\mathscr{F} m_{\mathscr{F}}\left|\mathbf{e}_{\lambda_{0}}^{*} \cdot \mathbf{D}\right| F m_{F}^{\prime}> \\
& +\Gamma_{F^{\prime} \mathscr{F}^{\prime}}^{\prime} \sum_{m_{\mathscr{F}^{\prime}}^{\prime}}<F m_{F^{\prime}}\left|\mathbf{e}_{\lambda_{0}} \cdot \mathbf{D}\right| \mathscr{F}^{\prime} m_{\mathscr{F}^{\prime}}^{\prime}> \\
& \left.<\mathscr{F}^{\prime} m_{\mathscr{F}^{\prime}}^{\prime}\left|\mathbf{e}_{\lambda_{0}}^{*} \cdot \mathbf{D}\right| F m_{F}^{\prime}\right\rangle \text {. }
\end{aligned}
$$

On trouvera dans la référence [3] la définition des opérateurs vectoriels hermitiques sans dimensions $\mathbf{S}$ et D dont les composantes $S^{(q)}$ et $D^{(q)}$ se transforment par rotation comme les harmoniques sphériques d'ordre 1 .

Nous introduisons les opérateurs $S_{p}^{(a)}$ et $D_{p}^{(a)}$

$$
S^{(\alpha)}=\sum_{p} S_{p}^{(q)} \quad D^{(q)}=\sum_{p} D_{p}^{(q)} \quad p=0, \pm 1
$$

Par définition l'un de ces opérateurs agissant sur un état $\mid F, m_{E}>$ augmente $F$ de $p$ unités : $S_{p}^{(a)}$ n'a d'éléments de matrice qu'entre l'état $\mid F, m_{k}>$ et l'état $\mid F+p, m_{F}+q>; D_{p}^{(a)}$ n'a 'd'éléments de matrice qu'entre l'état $\mid F, m_{F}>$ et l'état $\mid \mathcal{F}+p$, $m_{\mathscr{F}}+q>$.

Les opérateurs vectoriels $\mathbf{S}_{p}$ et $\mathbf{D}_{p}$ se transforment donc par rotation comme les harmoniques sphériques d'ordre 1.

L'application du théorème de Wigner-Eckart à $\mathbf{S}_{p}^{(a)}$ et $\mathbf{D}_{p}^{(a)}$ permet d'écrire

$<S_{1 / 2} J_{z}^{\prime}\left|\mathbf{D}_{p}^{(q)}\right| P_{1 / 2} J_{z}>=\lambda<S_{1 / 2} J_{z}^{\prime}\left|\mathbf{S}_{\nu}^{(q)}\right| S_{1 / 2} J_{z}>$ et par suite (avec $F^{\prime} \neq F$ )

$$
\begin{aligned}
& C_{F m_{F}^{F}}^{F m_{F}^{F}}=\Gamma_{F \mathscr{F}}^{\prime} \sum_{m_{\mathscr{F}}}<F m_{F}\left|\mathbf{\epsilon}_{\lambda_{0}} \cdot \mathbf{D}_{0}\right| F^{\prime} m_{\mathscr{F}}><\mathscr{F} m_{\mathscr{F}}\left|\mathbf{e}_{\lambda_{0}}^{*} \cdot \mathbf{D}_{0}\right| F m_{F}^{\prime}> \\
& +\Gamma_{F \mathscr{F}^{\prime}}^{\prime} \sum_{m^{\prime} \mathscr{F}^{\prime}}<F m_{F}\left|\mathbf{e}_{\lambda_{0}} \cdot \mathbf{D}_{ \pm 1}\right| \mathscr{F}^{\prime} m^{\prime} \mathscr{F}^{\prime}><\mathscr{F}^{\prime} m_{\mathscr{F}^{\prime}}^{\prime}\left|\mathbf{e}_{\lambda_{0}^{*}}^{*} \cdot \mathbf{D}_{ \pm 1}\right| F m_{F}^{\prime}> \\
& =\lambda^{2} \Gamma_{F F}^{\prime} \sum_{m_{F^{\prime}}}<F m_{F}\left|\mathbf{e}_{\lambda_{0}} \cdot \mathbf{S}_{0}\right| F m_{F}^{\prime \prime}><F m_{F}^{\prime \prime}\left|\mathbf{e}_{\lambda_{0}}^{*} \cdot \mathbf{S}_{0}\right| F m_{F}^{\prime}> \\
& \left.\left.+\lambda^{2} \Gamma_{F \mathcal{F}^{\prime}}^{\prime} \sum_{m_{-F^{\prime}}} \sum<F m_{F \mid}\left|\mathbf{e}_{\lambda_{0}} \cdot \mathbf{S}_{ \pm 1}\right| F^{\prime} m_{F^{\prime}}^{\prime \prime}\right\rangle<F^{\prime} m_{F^{\prime}}^{\prime \prime}\left|\mathbf{e}_{\lambda_{0}}^{*} \cdot \mathbf{S}_{ \pm 1}\right| F m_{\boldsymbol{F}}^{\prime}\right\rangle
\end{aligned}
$$


tenant compte des règles de sélection des transitions induites par les opérateurs $\mathbf{S}_{p}$

Comme

$$
\begin{aligned}
& C_{F m_{F}^{\prime}}^{F m_{F}}=\lambda^{2} \Gamma_{F \mathcal{F}}^{\prime}<F m_{F}\left|\mathbf{e}_{\lambda_{0}} \cdot \mathbf{S}_{\mathbf{0}}\left(e_{\lambda_{0}^{*}}^{*} \cdot \mathbf{S}_{\mathbf{0}}\right)\right| F m_{F}^{\prime}> \\
& +\lambda^{2} \Gamma_{F F^{\prime}}^{\prime}<F m_{F}\left|\mathbf{e}_{\lambda_{\theta}} \cdot \mathbf{S}_{ \pm 1}\left(\mathbf{e}_{\lambda_{\theta}^{*}}^{*} \cdot \mathbf{S}_{ \pm 1}\right)\right| F m_{F}^{\prime}>\text {. } \\
& <F m_{F}\left|S_{0}^{(q)}\right| F m_{F}^{\prime}>=g_{F}^{\prime}<F m_{F}\left|F^{(a)}\right| F m_{F}^{\prime}> \\
& C_{F m_{F}^{\prime}}^{F m_{F}}=\lambda^{2} \Gamma_{F F^{\prime}}^{\prime}<F m_{F}\left|\mathbf{e}_{\lambda_{0}} \cdot \mathbf{S}\left(\mathbf{e}_{\lambda_{0}^{*}}^{*} \cdot \mathbf{S}\right)\right| F m_{F}^{\prime}> \\
& +\lambda^{2} g_{F}^{\prime}\left(\Gamma_{F \mathcal{F}}^{\prime}-\Gamma_{F \mathcal{F}^{\prime}}^{\prime}\right)<F m_{F}\left|\mathbf{e}_{\lambda_{0}} \cdot \mathbf{F}\left(\mathbf{e}_{\lambda_{0}}^{*} \cdot \mathbf{F}\right)\right| F m_{F}^{\prime}>\text {. }
\end{aligned}
$$

Pour un faisceau de lumière naturelle dirigé suivant $O x$ (superposition incohérente de lumière $\pi\left(\mathbf{e}_{\lambda}=\mathbf{u}_{z}\right)$ et de lumière $\sigma\left(\mathbf{e}_{\lambda}=\mathbf{u}_{y}\right)$, nous obtenons

$$
\begin{aligned}
C_{F m_{F}^{\prime}}^{F m_{F}} & =\frac{\lambda^{2}}{4} \Gamma_{F \mathcal{F}^{\prime}}^{\prime}<F m_{F}\left|P_{F}\right| F m_{F}^{\prime}> \\
& +\frac{\lambda^{2}}{2} g_{F}^{\prime 2}\left(\Gamma_{F \mathcal{F}}^{\prime}-\Gamma_{F \mathcal{F}^{\prime}}^{\prime}\right)<F m_{F}\left|F^{2}-F_{x}^{2}\right| F m_{F}^{\prime}>
\end{aligned}
$$

et

$$
\begin{aligned}
& L_{\mathrm{A}}=\frac{\lambda^{2}}{8}\left(\Gamma_{F_{+} \mathcal{F}_{-}}^{\prime}+\Gamma_{F_{-} \mathcal{F}_{+}}^{\prime}\right)+\frac{\lambda^{2}}{8}\left(\Gamma_{F_{+} \mathcal{F}_{-}}^{\prime}-\Gamma_{F-\mathcal{F}_{+}}^{\prime}\right) \operatorname{Tr}\left(P_{F_{+}}-P_{F_{-}}\right) \\
& +\frac{\lambda^{2}}{2} g_{F}^{\prime 2}\left(\Gamma_{F+F_{+}}^{\prime}-\Gamma_{F+F_{-}}^{\prime}\right) \operatorname{Tr} P_{F_{+}}\left(\mathbf{F}^{2}-F_{x}^{2}\right) \\
& +\frac{\lambda^{2}}{2} g_{F}^{\prime 2}\left(\Gamma_{F-F_{-}}^{\prime}-\Gamma_{F-F_{+}}^{\prime}\right) \operatorname{Tr} P_{F-}\left(F^{2}-F_{x}^{2}\right) \text {. }
\end{aligned}
$$

Posant :

on en déduit :

$$
k=\frac{\lambda^{2}}{16} \cdot \frac{i_{\mathbf{2}}^{\prime \prime}}{\Gamma_{F+\mathfrak{F}-}^{\prime}},
$$

$$
\begin{aligned}
L_{\mathbf{A}} & =k\left(i_{2}^{\prime \prime}+i_{1}^{\prime \prime}+i_{2}^{\prime}+i_{1}^{\prime}\right) \\
& +k\left(i_{2}^{\prime \prime}+i_{2}^{\prime}-i_{1}^{\prime \prime}-i_{1}^{\prime}\right)<P_{F+}-P_{F-}> \\
& +8 k\left(i_{2}^{\prime}-i_{2}^{\prime \prime}\right)<P_{F+}\left[g_{F}^{\prime 2}\left(\mathbf{F}^{2}-F_{x}^{2}\right)-\frac{1}{4}\right]> \\
& +8 k\left(i_{1}^{\prime}-i_{1}^{\prime \prime}\right)<P_{F-}\left[g_{F}^{\prime 2}\left(\mathbf{F}^{2}-F_{x}^{2}\right)-\frac{1}{4}\right]>.
\end{aligned}
$$

Pour une excitation en lumière $\pi(o u \sigma)$ on obtient une expression identique à celle ci-dessus en remplaçant seulement $\mathbf{F}^{2}-F_{x}^{2}$ par $2 F_{z}^{2}$ (ou par $2 F_{y}^{2}$ ).

Remarque: Ceci permet de prévoir l'aspect différent du transitoire pendant le temps $T_{2}$ suivant que $\mathrm{F}^{d}$ est polarisé en $\pi$ (détecte $\left\langle F_{z}^{2}\right\rangle$ ) ou en $\sigma$ (détecte $\left\langle F_{x}^{2}\right\rangle$ ) : sur un intervalle de temps long devant $1 / 2 \omega_{0},<\bar{F}_{x}^{2}>=\frac{1}{2}<F^{2}-F_{z}^{2}>$.

Manuscrit reçu le 22 décembre 1965.

\section{BIBLIOGRAPHIE}

[1] Kastler (A.), J. Physique Rad., 1950, 11, 255.

[2] Brosser (J.), dans Optique et Électronique Quantique, édité par De Witt, Blandin, CohenTannoudji, Gordon and Breach, 1964.

[3] Bouchiat (M. A.), J. Physique, 1965, 26, 417.]

[4] Franzen (W.), Phys. Res., 1959, 115, 850.

[5] Bouchiat (M. A.), Thèse, Paris, 1964; Publications Scientifiques et Techniques du Ministère de l'Air, N. T. 146, 1965.

[6] Bouchiat (M. A.), J. Physique, 1963, 24, 379 et 611.

[7] Grossetête (F.), J. Physique, 1964, 25, 383.

[8] Grossetête (F.), A paraître.

[9] Référence [5], page 121.

[10] Bouchiat (M. A.) et Brossel (J.), C. R. Acad. Sc., 1963, 257, 2825.
[11] Référence [5], pages 92 et 97.

[12] Geschwind (S.), Devilin (G. E.), Cohen (R. L.) et Chinn (S. R.), Phys. Rev., 1965, 137, A 1087. Nous avons utilisé l'extracteur de signaux noyés dans le bruit, modèle ND-800, 1024 canaux (“Enhancetron »- Nuclear Data).

[13] Brewer (R. G.), J. O. S. A., 1962, 52, 832 et référence [5]; p. 113.

[14] Bender (P. L.), Beaty (F. C.) et Chi (A. R.), Phys. Res., 1958, 112, 450.

[15] Grossetête (F.), C. R. Acad. Sc., 1964, 259, 3211.

[16] Taylor (J. B.) et Langmuir (I.), Phys. Rev., 1937, $51,753$.

[17] Mocs (H. W.) et Sands (R. H.), Phys. Rev., 1964, 135, A 591 . 